\title{
Multifield variational formulations of diffusion initial boundary value problems
}

\author{
Jorge de Anda Salazar . Thomas Heuzé* • Laurent \\ Stainier
}

\begin{abstract}
We present two multifield and one single-field variational principles for the Initial Boundary Value Problem of diffusion. Chemical potential and concentration appear as conjugate variables in the multifield formulations. The main importance of the proposed formulations is the approach used to generate the variational principles, where the framework of Generalized Standard Materials is used for constitutive laws while natural boundary conditions and the balance of mass are used as constraints of the optimization problem. This approach allows to derive such principles for multiphysic problems in a generic manner. A detailed derivation and analysis of the formulations is presented, where it can be seen their equivalence with the most common strong and weak forms of the problem using Fick's laws along with the logarithmic mass action law. From the stationarity condition with respect to the mass flux of the initially proposed functional, two main relations are identified. First, the chemical potential appears as the opposite of the Lagrange multiplier that allows to enforce the balance of mass and natural boundary conditions. Second, a conjugate relation is found for a given substance between its mass flux and the opposite of the gradient of its chemical potential. Furthermore, to reduce the number of variables of the initial variational principle, a field reduction is applied, reaching the model presented by [1] for Fickean diffusion. Nevertheless, the aforementioned relations cannot be derived from the reduced model. Finally, a numerical implementation is presented for completeness where we compare the performance of the proposed formulations against the usual weak form.
\end{abstract}

\footnotetext{
* Corresponding author.

Jorge de Anda Salazar

Institut de Recherche en Génie Civil et Mécanique (GeM, UMR 6183 CNRS/ECN/UN)

École Centrale Nantes, 1 rue de la Noë, BP 92101, F-44321 Nantes, France

E-mail: Jorge.De-Anda-Salazar@ec-nantes.fr

Thomas Heuzé

Institut de Recherche en Génie Civil et Mécanique (GeM, UMR 6183 CNRS/ECN/UN)

École Centrale Nantes, 1 rue de la Noë, BP 92101, F-44321 Nantes, France

Corresponding author

E-mail: thomas.heuze@ec-nantes.fr

Laurent Stainier

Institut de Recherche en Génie Civil et Mécanique (GeM, UMR 6183 CNRS/ECN/UN)

École Centrale Nantes, 1 rue de la Noë, BP 92101, F-44321 Nantes, France

E-mail: laurent.stainier@ec-nantes.fr
} 
Keywords Multifield diffusion · Variational formulation · Fick's laws · Logarithmic mass action law · Coupled problem

\section{Introduction}

In his seminal paper [2], Adolf Fick established the mathematical structure of the diffusion process, giving rise to what is called Fick's laws. This set of equations, which uses the concentration $c$ and the mass flux $\boldsymbol{j}$ as state variables, constitutes the most general way to model diffusion. Nevertheless, a wide variety of models can be derived to describe more specialized diffusion processes. Some of these more specialized processes of diffusion consist in redefining the constitutive law for the mass flux $\boldsymbol{j}$, which can be justified by the introduction of the chemical potential $\mu$.

It is a common practice when using Fick's laws to solve for the concentration $c$ and if needed compute the chemical potential $\mu$ afterwards through a constitutive law. We refer to this procedure as a post-process relation. The mass action laws [3] are empirical relations which can be used for such purpose. However, such post-process relation describes a system where the chemical potential does not affect the behavior of the system. The importance of the chemical potential as a state variable was initially highlighted by J. W. Gibbs [4]. More recently, this approach is supported by different authors, e.g. [5-7]. Lars Onsager took the mathematical description of diffusion a step further, and suggested that the chemical potential can be understood as the driving force [8] for transport in irreversible processes. In addition, concepts like the principle of maximum dissipation [8-11], and thermodynamic fields [12-14] play an important role in the thermodynamics of irreversible processes. Previous attempts at variational formulations of thermodynamic equations can found in $[15,16]$.

The aim of this work is to present two multifield and one single-field variational principles for the initial boundary value problem of diffusion. The variational principles proposed in this paper use the framework of Generalized Standard Materials [17] for the constitutive laws. Balance laws and natural boundary conditions are imposed as constraints into the system. As a result, the chemical potential is identified as the opposite of the Lagrange multiplier that allows to enforce the balance of mass and the natural boundary conditions. Plus, the concentration and chemical potential become conjugate fields, in essence, thermodynamic fields. Likewise, the proposed variational principles also define the mass flux $\boldsymbol{j}$ as a conjugate field of the opposite of the gradient of the chemical potential $-\nabla \mu$ through the conjugate function $\chi^{*}[18]$. The conjugate function $\chi^{*}$ is identified as the dissipation potential needed to enforce the second principle of thermodynamics in the model. The latter highly contrasts with the explicit dependence defined by Fick's first law $\boldsymbol{j}(c)$.

The variational formulation could be applied to any multiphysic problem which has a gradient flow mathematical structure. Examples are mechanics, heat transfer, diffusion, electricity, magnetism, and any combination of these. Thus, the multifield modelling of diffusion obtained with the proposed variational principles opens the path to explore multiphysic problems. The variational principles can be generalized since the specifics of each physics involved are handled by the functional and the potentials. A parallel formulation could be made with the $\mathrm{Hu}$-Washizu-De Veubeke variational principle in mechanics [19], and its connection with Hellinger-Reissner, or the minimum potential energy [20], [21], $[22]$. In addition, the initial variational principle is formulated as a constrained optimization problem. Moreover, the variables specific to each branch of physics are described as energetically-conjugated and can be identified as flux-like, force-like or displacement-like fields. All the former allows to define the coupling between the different physics under the definition of the potentials. 
From the variational perspective, the existence of a unique solution is linked to a unique stationary point characterized by the convexity/concavity of the functional with respect to variations. Systems where material properties depend on the fields can be modeled, provided these dependencies maintain the respective convexity/concavity of the stationary point. Nevertheless, the consistency of the variations may be lost once the functional is transformed from continuum to discrete form.

Furthermore, the existence of a variational principle is of interest not just from a continuum viewpoint but also from a numerical point of view. The computational aspects gained begin with the matrix form of the problem which is symmetric. Therefore, several techniques can be explored to improve the computational cost of the solution. Moreover, since boundary, constitutive and balance equations are embedded in the functional, the possibility to derive different -not necessarily monolithic*- numerical strategies which have a mathematical representation and are consistent with the continuum model is possible.

The outline of the paper is as follows: a detailed derivation and analysis of the principles is presented in sections 2 and 3 , where their respective equivalence with the most common strong and weak forms of the problem using Fick's laws and the logarithmic mass action law are illustrated. Moreover, by applying a field reduction on the initially derived multifield variational formulation, we reach the model already presented by [1]. Nevertheless, the identification of the chemical potential as the opposite of a Lagrange multiplier and the conjugate relation between mass flux and the opposite gradient of the chemical potential cannot be derived from the reduced model. In section 5 the numerical counterpart of the formulation is shown, where we analyze the implications of moving from the continuum to the discrete setting. In section 6.1 we solve $1 \mathrm{D}$ and $2 \mathrm{D}$ problems numerically starting from the presented variational formulation. Finally, in section 7 the conclusions and perspectives of the work are presented.

\section{Diffusion initial boundary value problem formulations}

\subsection{Strong form}

We are interested in the problem of diffusion of a given species within a continuous medium, occupying a domain $\Omega$. Let us assume that the local state at a material point $\boldsymbol{X} \in \Omega$ can be described by the concentration $c$ (expressed as volume fraction, molar fraction, or any other convenient equivalent) of the diffusing species and its corresponding chemical potential $\mu$. On the one hand, the evolution of the diffusing species is described by the mass balance equation ${ }^{\dagger}$.

$$
\dot{c}+\operatorname{div} \boldsymbol{j}=r \quad \forall \boldsymbol{X} \in \Omega \quad \text { Fick's second law }
$$

where $\boldsymbol{j}$ is the species flux and $r$ the local source. On the other hand, the constitutive laws of the system are described by

$$
\begin{array}{lll}
\boldsymbol{j}=-D \boldsymbol{\nabla} c & \forall \boldsymbol{X} \in \Omega & \text { Fick's first law } \\
\mu=\mu^{\ominus}+R T \ln \left(\frac{c}{c^{\ominus}}\right) & \forall \boldsymbol{X} \in \Omega & \text { Logarithmic mass action law }
\end{array}
$$

\footnotetext{
*We refer as monolithic numerical strategies, to a discrete system of equations on which all the variables are solved synchronously.

${ }^{\dagger}$ For the purpose of the presentation, we focus on the specific problem below, but the same ideas hold to any more general modelling.
} 
where $D$ is the diffusion coefficient (we assume isotropy for simplicity), $\mu^{\ominus}$ and $c^{\ominus}$ the corresponding reference values for the chemical potential and concentration respectively, $R$ is the universal gas constant and $T$ represents the temperature at which the process is taking place. The above equations are supplemented with boundary conditions: the outward flux $\bar{j}$ is imposed on the part $\partial_{j} \Omega$ of the boundary

$$
\boldsymbol{j} \cdot \boldsymbol{n}=\bar{j} \quad \forall \boldsymbol{X} \in \partial_{j} \Omega
$$

where $\boldsymbol{n}$ is the outward unit normal to the boundary, while on the remainder of the boundary $\partial_{\mu} \Omega=\partial \Omega \backslash \partial_{j} \Omega$, the chemical potential $\bar{\mu}$ (or equivalently the concentration $\bar{c}$ ) is imposed,

$$
\mu(c)=\bar{\mu} \quad \forall \boldsymbol{X} \in \partial_{\mu} \Omega .
$$

Finally, we impose the initial condition

$$
c(t=0)=c_{0} \quad \forall \boldsymbol{X} \in \Omega
$$

where for simplicity we consider $c_{0}$ to be constant within the domain $\Omega$. For simplicity here, we consider the initial conditions of the chemical potential and the concentration as equal to their reference values (2b), i.e., $\mu_{0}=\mu^{\ominus}$ and $c_{0}=c^{\ominus}$. These equations constitute the strong form of the diffusion initial boundary value problem.

It is usual to define the diffusion problem using only Fick's laws (2a) and (1) to solve for $\boldsymbol{j}$ and $c$, whereas a mass-action law (2b) is imposed after solving for $c$ to obtain $\mu$. This post-process relation between variables defines an explicit dependence of the form $\mu(c)$.

\subsection{Weak form}

The equivalent weak formulation of the above diffusion initial boundary value problem can be stated as

$$
\int_{\Omega}\left(\frac{\partial c}{\partial t} \delta c+D \nabla c \cdot \nabla \delta c\right) d V+\int_{\partial \Omega_{j}} \bar{j} \delta c d S=0 \quad \forall \delta c \in \mathcal{V}_{c}^{0}
$$

where $\delta c$ is a test function and $\mathcal{V}_{c}^{0}$ is its corresponding homogeneous space. Equation (6) encompasses Fick's laws. Again as with the strong form, a post-process relation is used to obtain $\mu$ through the mass action law $(2 \mathrm{~b})$. The admissible solution space is defined as follows

$$
\mathcal{V}_{c}=\left\{c \in H^{1}(\Omega) \mid c=\bar{c} \quad \forall \boldsymbol{X} \in \partial_{c} \Omega\right\}
$$

where $\bar{c}$ is the prescribed concentration on $\partial \Omega_{c}$ and $H^{1}(\Omega)$ stands for the Sobolev space [23]. As it is for the strong and the weak forms of the problem, often, the constitutive law (2b) is omitted. The latter being justified by the locality and the explicit dependence of $\mu$ with respect to $c$.

\section{Variational principle}

A mathematical model relies on three main elements, state variables, their relations and constraints. To construct a variational formulation the above items can be related to conjugate variables, potentials plus balance laws and associated boundary conditions respectively. 
3.1 Onsager's energy rate

Let's start by using Onsager's energy rate [9]

$$
\Pi_{0}[\dot{c}, \boldsymbol{j}]:=\int_{\tau}\left[\int_{\Omega}\left(\dot{G}(c)+\chi^{*}(\boldsymbol{j}, c)\right) d V+\int_{\partial_{\mu} \Omega} \bar{\mu}(\boldsymbol{j} \cdot \boldsymbol{n}) d S\right] d t
$$

with $G$ and $\chi^{*}$ as the internal energy and the dual dissipation potential, $\boldsymbol{j}$ the mass flux, $\boldsymbol{n}$ the outward unit normal and $\bar{\mu}$ a known value of the chemical potential defined at the Dirichlet boundary $\partial_{\mu} \Omega$. This energy-like representation of the system has also been used by many other authors [24-28, e.g.]. Since the internal energy $G$ is a state function, the first part of integral (8) only depends on the initial and final states. The dissipation potential $\chi^{*}$ depends on the whole history of the field $\boldsymbol{j}$ and $c$. Thus, in general, the integral $\Pi_{0}$ depends on the history of fields $c(\boldsymbol{X} ; t)$ and $\boldsymbol{j}(X ; t)$ : it is a path-dependent quantity.

The evolution of the system is obtained by optimizing Onsager's energy rate (8). However, we want to enforce this optimum under the constraint of the mass balance (1) and Neumann boundary condition (3). This leads to a constrained optimization problem. Thus, the primal problem to solve becomes

$$
\{\dot{c}, \boldsymbol{j}\}=\arg \inf _{\dot{c}, \boldsymbol{j}} \Pi_{0} \quad \text { subject to } \quad \begin{cases}\dot{c}+\boldsymbol{\nabla} \cdot \boldsymbol{j}-r=0 & \forall \boldsymbol{X} \in \Omega \\ \bar{j}-\boldsymbol{j} \cdot \boldsymbol{n}=0 & \forall \boldsymbol{X} \in \partial_{j} \Omega\end{cases}
$$

By means of the Lagrange multiplier method we can define the following functional

$$
\begin{aligned}
\Pi_{\lambda}\left[\dot{c}, \lambda_{1}, \lambda_{2}, \boldsymbol{j}\right] & :=\int_{\tau}\left[\int_{\Omega}\left(\dot{G}+\chi^{*}+\lambda_{1}(\dot{c}+\nabla \cdot \boldsymbol{j}-r)\right) d V\right. \\
& \left.+\int_{\partial_{\mu} \Omega} \bar{\mu}(\boldsymbol{j} \cdot \boldsymbol{n}) d S+\int_{\partial_{j} \Omega}\left(\lambda_{2}(\bar{j}-\boldsymbol{j} \cdot \boldsymbol{n})\right) d S\right] d t
\end{aligned}
$$

where $\lambda_{1}$ and $\lambda_{2}$ are the corresponding Lagrange multipliers.

\subsubsection{Optimality conditions}

The optimality conditions of the constrained functional $\Pi_{\lambda}$ are defined taking its variations.

First variations : The first variations of the functional should vanish since we look for a stationary point of the functional.

Variation with respect to $j$ : The variation with respect to $j$ of $\Pi_{\lambda}$ leads to

$$
<D_{j} \Pi_{\lambda}, \delta \boldsymbol{j}>\equiv 0 \quad \Rightarrow \quad \begin{cases}\lambda_{1}=-\bar{\mu} & \forall \boldsymbol{X} \in \partial_{\mu} \Omega \\ \lambda_{1}=\lambda_{2} & \forall \boldsymbol{X} \in \partial_{j} \Omega \\ \nabla \lambda_{1}=\frac{d \chi^{*}}{d \boldsymbol{j}} & \forall \boldsymbol{X} \in \Omega\end{cases}
$$

Several interesting facts can be observed. First, (10.a) indicates that the Lagrange multiplier $\lambda_{1}$ is equal to the opposite of the chemical potential at the Dirichlet boundary. Second, equation (10.b) shows both Lagrange multipliers are actually the same variable. Third, (10.c) states that the opposite of the gradient of the chemical potential, in essence, the chemical field $-\nabla \mu:=\boldsymbol{g}$ is the dual variable 
of the mass flux $\boldsymbol{j}$ through the dual dissipation function $\chi^{*}$.

Variation with respect to $\dot{c}:$ The variation of $\Pi_{\lambda}$ with respect to $\dot{c}$ leads to

$$
<D_{\dot{c}} \Pi_{\lambda}, \delta \dot{c}>\equiv 0 \quad \Rightarrow \quad\left\{-\lambda_{1}=\frac{d G}{d c} \quad \forall \boldsymbol{X} \in \Omega\right.
$$

The internal energy is the conjugate function which defines the conjugate relation between the chemical potential and the concentration.

Variation with respect to $\mu$ : The variation with respect to the chemical potential, i.e. the opposite of the Lagrange multiplier yields

$$
<D_{\mu} \Pi_{\lambda}, \delta \mu>\equiv 0 \quad \Rightarrow \quad \begin{cases}\dot{c}=-\boldsymbol{\nabla} \cdot \boldsymbol{j}+r & \forall \boldsymbol{X} \in \Omega \\ \bar{j}=\boldsymbol{j} \cdot \boldsymbol{n} & \forall \boldsymbol{X} \in \partial_{j} \Omega\end{cases}
$$

The use of the opposite of the chemical potential as a Lagrange multiplier dates back to at least as far as [29]. The stationary conditions of the functional $\Pi_{\lambda}$ yield the strong form of the equations presented in section 2.1. More details about the derivation of first variations of the functional $\Pi_{\lambda}$ are presented in appendix A.

Second variations : The second variations allow to determine the type of the stationary point of the functional.

Variation with respect to $\dot{c}$ : The second variation of (11) results in

$<D_{\dot{c}}^{2} \Pi_{\lambda}, \delta \dot{c}^{2}>=\int_{\tau}\left[\int_{\Omega} \frac{d^{2} G}{d c^{2}} d V\right] \delta \dot{c}^{2} d t \Rightarrow \frac{d^{2} G}{d c^{2}}\left\{\begin{array}{l}>0, \Pi_{\lambda} \text { admits a minimum with respect to } \dot{c} \\ <0, \Pi_{\lambda} \text { admits a maximum with respect to } \dot{c} \\ =0, \Pi_{\lambda} \text { is neutral with respect to } \dot{c}\end{array}\right.$

As shown above the optimal condition for $\dot{c}$ depends on the second derivative of the internal energy. The definition of $G$ is thus of great importance.

Variation with respect to $\mu$ : Due to strong duality properties as stated by $[30, \S 5.2 .3]$, the chemical potential, must have an opposite definition compared to the concentration. Thus, we expect the stationary point of the functional to be a saddle point.

Variation with respect to $\boldsymbol{j}$ : Finally the second variation of $\Pi_{\lambda}$ with respect to $\boldsymbol{j}$, resembles the second variation with respect to $\dot{c}$, but now involves the dissipation potential instead of the internal energy

$$
<D_{\boldsymbol{j}}^{2} \Pi_{\lambda}, \delta \boldsymbol{j} \delta \boldsymbol{j}>=\int_{\tau}\left[\int_{\Omega} \frac{d^{2} \chi^{*}}{d \boldsymbol{j}^{2}} d V\right] \delta \boldsymbol{j} \delta \boldsymbol{j} d t
$$

However, thermodynamic consistency imposes the convexity of $\chi^{*}$ as it will be shown in section 4 . Therefore, the second derivatives of $\chi^{*}$ are positive, this gives a minimum condition on $\boldsymbol{j}$. This optimal condition on $j$ is a way to ensure that the second principle of thermodynamics is satisfied.

\subsection{Variational formulations}

The functional (9) satisfies our requirements to model the multifield chemical diffusion problem. It embeds the mass balance and constitutive laws, namely Fick's first law and mass action law into the functional and the different potential functions $G$ and $\chi^{*}$, respectively. Three variational formulations can be developed according to the number of fields the functional depends on. 


\subsubsection{3-Field variational form}

Under the assumption that $G(c)$ is convex, replacing the Lagrange multipliers and applying the divergence theorem to the term containing $\nabla \cdot \boldsymbol{j}$ in the functional $\Pi_{\lambda}$ (9) yields the 3-Field variational form shown below.

$$
\begin{gathered}
\frac{\text { 3-Field variational form : }}{\{\boldsymbol{j}, \dot{c}, \mu\}=\arg \inf _{\dot{c}, \boldsymbol{j}} \sup _{\mu} \Pi_{3}[\boldsymbol{j}, \dot{c}, \mu]} \\
\Pi_{3}[\dot{c}, \boldsymbol{j}, \mu]=\int_{\tau}\left[\int_{\Omega}\left(\dot{G}-\mu \dot{c}+\chi^{*}(c, \boldsymbol{j})-\boldsymbol{g} \cdot \boldsymbol{j}+\mu r\right) d V-\int_{\partial_{j} \Omega} \mu \bar{j} d S\right] d t
\end{gathered}
$$

\subsubsection{2-Field variational form}

A 2-Field variational form is obtained by replacing the mass flux by the chemical field. Applying the Legendre-Fenchel transform

$$
\chi(\boldsymbol{g}, c)=\sup _{\boldsymbol{j}}\left\{\boldsymbol{g} \cdot \boldsymbol{j}-\chi^{*}(\boldsymbol{j}, c)\right\}
$$

yields the primal dissipation potential that depends on the concentration and the chemical field. Using the above substitution leads to the functional

\section{2-Field variational form :}

$$
\begin{gathered}
\{\dot{c}, \mu\}=\arg \inf _{\dot{c}} \sup _{\mu} \Pi_{2}[\dot{c}, \mu] \\
\Pi_{2}[\dot{c}, \mu]=\int_{\tau}\left[\int_{\Omega}(\dot{G}(c)-\mu \dot{c}-\chi(\boldsymbol{g}, c)+\mu r) d V-\int_{\partial_{j} \Omega} \mu \bar{j} d S\right] d t
\end{gathered}
$$

\subsubsection{1-Field variational form}

A further field reduction can be made so that the optimization is performed with respect to the sole chemical potential. This leads to the 1-Field variational form. The Legendre-Fenchel transform is applied to the internal energy to define the following dual potential

$$
G^{*}(\mu)=\sup _{c}\{\mu c-G(c)\} \quad \rightarrow \quad \dot{G}^{*}(\mu)-\dot{\mu} c=\mu \dot{c}-\dot{G}(c)
$$

Substituting the internal energy rate by its dual counterpart yields

$$
\Pi_{1}[\mu]=\int_{\tau}\left[\int_{\Omega}\left(\dot{\mu} c-\dot{G}^{*}(\mu)-\chi(\boldsymbol{g}, c(\mu))+\mu r\right) d V-\int_{\partial_{j} \Omega} \mu \bar{j} d S\right] d t
$$

However, the functional above still contains the concentration field. If we express it in terms of the chemical potential, i.e. $c=\frac{d G^{*}}{d \mu}$ the first two terms cancel each other, therefore the remaining functional becomes

1-Field variational form :

$$
\begin{gathered}
\{\mu\}=\arg \sup _{\mu} \Pi_{1}[\mu] \\
\Pi_{1}[\mu]=\int_{\tau}\left[\int_{\Omega}(-\chi(\boldsymbol{g}, c(\mu))+\mu r) d V-\int_{\partial_{j} \Omega} \mu \bar{j} d S\right] d t
\end{gathered}
$$


3.3 Equivalence between strong and variational formulations

The equivalence between the strong form and the 3-Field variational formulation has been presented in section 3.1.1. Now, the field reduction leading to (15) is equivalent to the original strong form of the problem presented in section 2.1 while the reduced functional (17) is only equivalent to the steady case of the problem.

\subsubsection{2-Field variational form}

The variations applied to the 2-Field variational form presented in (15) are shown below.

Variation with respect to $\dot{c}$ : The first variation of $\Pi_{2}$ with respect to $\dot{c}$ gives the same result than that of $\Pi_{3}$, since this reduction does not involves the concentration.

$$
<D_{\dot{c}} \Pi_{2}, \delta \dot{c}>\equiv 0 \quad \quad \quad \quad\left\{\mu=\frac{d G}{d c} \quad \forall \boldsymbol{X} \in \Omega\right.
$$

Variation with respect to $\mu$ : This process requires an extra step, which is the use of the divergence theorem after applying the variation to the dissipation function. The outcome being

$$
<D_{\mu} \Pi_{2}, \delta \mu>\equiv 0 \quad \Rightarrow \quad \begin{cases}\dot{c}=-\nabla \cdot \frac{\partial \chi}{\partial \boldsymbol{g}}+r & \forall \boldsymbol{X} \in \Omega \\ \bar{j}=\frac{\partial \chi}{\partial \boldsymbol{g}} \cdot \boldsymbol{n} & \forall \boldsymbol{X} \in \partial_{j} \Omega\end{cases}
$$

Therefore, the field reduction removes the mass flux and replaces it by $\frac{\partial \chi}{\partial \boldsymbol{g}}$. Moreover, the identification of the chemical potential as a Lagrange multiplier is also lost. These changes however still keep the equivalence with the strong form if we define $\boldsymbol{j}:=\frac{\partial \chi}{\partial \boldsymbol{g}}$.

\subsubsection{1-Field variational form}

Variation with respect to $\mu$ : The variation of $\Pi_{1}(17)$ with respect to the chemical potential

$$
<D_{\mu} \Pi_{1}, \delta \mu>\equiv 0 \quad \Rightarrow \quad \begin{cases}\nabla \cdot \frac{\partial \chi}{\partial \boldsymbol{g}}=r & \forall \boldsymbol{X} \in \Omega \\ \bar{j}=\frac{\partial \chi}{\partial \boldsymbol{g}} \cdot \boldsymbol{n} & \forall \boldsymbol{X} \in \partial_{j} \Omega\end{cases}
$$

The above is equivalent with the strong form of the steady problem as long as $\chi$ does not have any dependence on $c$. In addition, we must define $c:=\frac{\partial G^{*}}{\partial \mu}$ and $j:=\frac{\partial \chi}{\partial \boldsymbol{g}}$ to retrieve the information of the state variables.

In the 3-Field formulation $\Pi_{3}$, we derived two important relations. First, the chemical potential is linked to a Lagrange multiplier enforcing the mass balance and Neumann boundary conditions. Second, the mass flux is a conjugate variable of the chemical field. The latter relations can not be derived from the 2-Field formulation $\Pi_{2}$. Nevertheless, the system described by both formulations is equivalent. Hence, the 2-Field formulation provides the best compromise between complexity and generality since it allows for treating transient diffusion problems with a minimum number of independent fields that are scalar quantities. In contrast, the 3-Field formulation would increase the computational cost in the discrete setting. Therefore, in the following we use the 2-Field as our main formulation. The formulation for $\Pi_{2}$ is similar to that obtained directly by [1] where they directly adapted the strong form of the problem to fit into a variational formulation, using a variational derivative. The advantage of our approach resides in the two relations mentioned above which are derived and provide further knowledge into the system. Furthermore, our approach allows to construct the functional for 
the variational principles using balance laws (as well as Neumann conditions) as constraints, instead of directly using the strong form of the system, which opens the path to generalize the approach to multiphysic problems.

\section{Specification of the material}

Generalized Standard Materials [17] are defined using two scalar functions, a thermodynamic potential $G$ plus a dissipation one $\chi$, which are both convex functions of their respective arguments, nonnegative $(G \geq 0, \chi \geq 0)$ and are equal to zero at their origin $(G(0)=0, \chi(\mathbf{0}, 0)=0)$. The internal energy $G$ and the dissipation potential $\chi$ define the constitutive laws of the system.

\subsection{The Linear model}

\subsubsection{Internal energy}

A linearized version of the logarithmic mass action law (2b) reads

$$
\mu=\mu_{0}+\frac{R T}{c_{0}}\left(c-c_{0}\right)
$$

Since $\mu$ is energetically-conjugate to $c$ through the internal energy $G(c)(11)$, the integral of (21) with respect to $c$ defines a quadratic potential $G(c)$.

$$
G(c)=\mu_{0} c+\frac{1}{2} \frac{R T}{c_{0}}\left(c-c_{0}\right)^{2} ; \quad \frac{d G}{d c}=\mu_{0}+\frac{R T}{c_{0}}\left(c-c_{0}\right) ; \quad \frac{d^{2} G}{d c^{2}}=\frac{R T}{c_{0}}
$$

\subsubsection{Dissipation potential}

Multiplying the residual of the mass balance by the chemical potential and integrating over the domain $\Omega$ we get the following energy balance

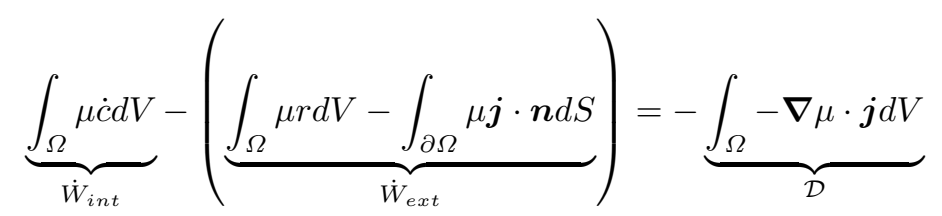

where the boundary term and the term on the right hand side have been obtained by using the divergence theorem. We can rewrite the above equation as

$$
\dot{W}_{\text {ext }}-\dot{W}_{\text {int }}=\mathcal{D} \geq 0
$$

where $\dot{W}_{\text {int }}, \dot{W}_{\text {ext }}$ and $\mathcal{D}$ denote the internal and external energy rates and the dissipation respectively. This equation is the same as that shown by [1, Eq. 18]. The difference between the external and internal energy rates of the system must be positive. This last statement imposes a condition on the dissipation $\mathcal{D}$ which should be non-negative. Provided the chemical field and the mass flux $\boldsymbol{j}$ are energetically-conjugate, the dissipation reads

$$
\mathcal{D}:=\int_{\Omega}-\nabla \mu \cdot \boldsymbol{j} d V=\int_{\Omega} \boldsymbol{j} \cdot \boldsymbol{g} d V=\int_{\Omega} \frac{\partial \chi}{\partial \boldsymbol{g}} \cdot \boldsymbol{g} d V \geq 0
$$


The inequality (24) appears as an entropy-like condition and it is automatically satisfied if $\chi$ is convex. An analog expression can also be written with the dual potential $\chi^{*}$. The simplest constitutive law satisfying (24) is a linear relation between the mass flux and the chemical field

$$
\boldsymbol{j}=D_{\mu} \boldsymbol{g}
$$

with $D_{\mu}>0$. Integrating with respect to $\boldsymbol{g}, \chi$ reads

$$
\chi(\boldsymbol{g}, c)=\frac{D_{\mu}}{2} \boldsymbol{g} \cdot \boldsymbol{g} ; \quad \frac{\partial \chi}{\partial \boldsymbol{g}}=D_{\mu} \boldsymbol{g} ; \quad \frac{\partial^{2} \chi}{\partial \boldsymbol{g}^{2}}=D_{\mu}
$$

The two potentials (22) and (26) yield linear state and complementary laws respectively and constitute what we call the Linear model of chemical diffusion. The model is useful for materials having linear properties, but also for non-linear materials undergoing small deviations.

\subsection{The Fickean model}

One way to describe non-linear materials is through the Fickean model obtained here.

\subsubsection{Internal energy}

Integrating the logarithmic mass action law $(2 \mathrm{~b})$ with respect to $c$ yields the corresponding internal energy:

$$
\begin{array}{rlrl}
G(c) & =\mu_{0} c+R T\left[c \ln \left(\frac{c}{c_{0}}\right)-c+c_{0}\right] ; & & \\
\frac{d G}{d c} & =\mu_{0}+R T \ln \left(\frac{c}{c_{0}}\right) ; & \frac{d^{2} G}{d c^{2}}=\frac{R T}{c}
\end{array}
$$

\subsubsection{The dissipation potential}

The constitutive law presented in [31] combined with (10.c) yields

$$
\boldsymbol{j}=-c \mathfrak{m} \nabla \mu=c \mathfrak{m} \boldsymbol{g} \equiv \frac{\partial \chi}{\partial \boldsymbol{g}}
$$

where $c$ is the concentration and $\mathfrak{m}$ is the mobility, both parameters are non-negative, thus this constitutive law is in agreement with (24). Using this relation, the obtained dissipation potential reads

$$
\chi(\boldsymbol{g}, c)=\frac{c \mathfrak{m}}{2} \boldsymbol{g} \cdot \boldsymbol{g} ; \quad \frac{\partial \chi}{\partial \boldsymbol{g}}=c \mathfrak{m} \boldsymbol{g} ; \quad \frac{\partial^{2} \chi}{\partial \boldsymbol{g}^{2}}=c \mathfrak{m}
$$

The potentials in (27) and (29) define the Fickean model. Indeed, the constitutive laws consist of equations (28) and (2b). Equation (28) is consistent with Fick's first law (2a), especially with its diffusion parameter $D$, as shown hereafter. 
4.3 Relation between the models and the diffusion parameter $D$

Fickean model : The diffusion parameter $D$ appearing in Fick's first law (2a) embeds information from both the logarithmic mass action law (2b) and the complementary law (28). The combination of both gives the constitutive laws for the chemical potential $\mu$ and the mass flux $\boldsymbol{j}$. Focusing on the Fickean model, starting from (28) we get

$$
\begin{aligned}
\boldsymbol{j} & =c \mathfrak{m} \boldsymbol{g} & & (\boldsymbol{g}:=-\nabla \mu) \\
& =-c \mathfrak{m} \nabla \mu & & \\
& =-c \mathfrak{m} \frac{R T}{c} \nabla c & & (D \equiv \mathfrak{m} R T) \\
& =-D \nabla c & &
\end{aligned}
$$

The above process shows the equivalence between the diffusion parameter $D$ and the terms $\mathfrak{m} R T$. Since $R:=N_{A} k_{B} \ddagger$ is a universal parameter -independent of the substance being described- and we are considering $T$ as constant, the information of the material is enclosed in $D$ as in $\mathfrak{m}$ through the equivalence $D \equiv \mathfrak{m} R T$. The parameter $D$ encompasses the information from the parameters in the equations (28) and (2b). This relation relates to the Einstein-Smoluchowski relation, $D \equiv \mathfrak{m} k_{B} T$ [32].

Linear model: The relation of $D$ is not exclusive to the Fickean model, it can also be related to the Linear model starting from (25) as follows.

$$
\begin{aligned}
\boldsymbol{j} & =D_{\mu} \boldsymbol{g} & & (\boldsymbol{g}:=-\nabla \mu) \\
& =-D_{\mu} \nabla \mu & & \\
& =-D_{\mu} \frac{R T}{c_{0}} \nabla c & & \left(D \equiv D_{\mu} \frac{R T}{c_{0}}\right) \\
& =-D \nabla c & &
\end{aligned}
$$

In this model, once again $D$ contains information from the involved equations (22) and (25). However, it also contains an extra dependency with the initial values of the concentration $c_{0}$, whereas on the Fickean model, it is completely independent of any field.

\subsection{Constitutive models}

As a summary, we have set up two different constitutive models. A non-linear one that consists of the logarithmic mass action law (2b), derived from the internal energy (27.b) and Fick's first law for the dissipation rewritten with the chemical field $\boldsymbol{g}$ in (28). This model is referred to as the Fickean model.

\footnotetext{
${ }^{\ddagger} N_{A}$ : Avogadro constant, $k_{B}$ : Boltzmann constant
} 
Fickean model:

$\underline{\text { Internal energy }}$

$$
\begin{aligned}
G(c) & =\mu_{0} c+R T\left[c \ln \left(\frac{c}{c_{0}}\right)-c+c_{0}\right] \\
\frac{d G}{d c} & =\mu_{0}+R T \ln \left(\frac{c}{c_{0}}\right) \\
\frac{d^{2} G}{d c^{2}} & =\frac{R T}{c}
\end{aligned}
$$

The second model is the linear counterpart of the Fickean model, and we refer to it as the Linear model.

\section{Linear model:}

$\underline{\text { Internal energy }}$

$$
\begin{aligned}
G(c) & =\mu_{0} c+\frac{1}{2} \frac{R T}{c_{0}}\left(c-c_{0}\right)^{2} \\
\frac{d G}{d c} & =\mu_{0}+\frac{R T}{c_{0}}\left(c-c_{0}\right) \\
\frac{d^{2} G}{d c^{2}} & =\frac{R T}{c_{0}}
\end{aligned}
$$

Dissipation potential

$$
\begin{aligned}
\chi(\boldsymbol{g}, c) & =\frac{1}{2} \frac{D}{R T} c \boldsymbol{g} \cdot \boldsymbol{g} \\
\frac{\partial \chi}{\partial \boldsymbol{g}} & =\frac{D}{R T} c \boldsymbol{g} \\
\frac{\partial^{2} \chi}{\partial \boldsymbol{g}^{2}} & =\frac{D}{R T} c
\end{aligned}
$$

Dissipation potential

$$
\begin{aligned}
\chi(\boldsymbol{g}, c) & =\frac{1}{2} \frac{D}{R T} c_{0} \boldsymbol{g} \cdot \boldsymbol{g} \\
\frac{\partial \chi}{\partial \boldsymbol{g}} & =\frac{D}{R T} c_{0} \boldsymbol{g} \\
\frac{\partial^{2} \chi}{\partial \boldsymbol{g}^{2}} & =\frac{D}{R T} c_{0}
\end{aligned}
$$

Both models above satisfy the convexity constraints imposed by the modeling on the internal energy and the dissipation Potential. Therefore, we know a unique solution exists which is linked to these unique stationary points. It may happen that with other models the considered functional may loose convexity due to coupling effects. The coupling effects come from the dependency of coefficients in the dissipation potential, as for instance through the concentration. Moreover, despite the convexity constraint can be satisfied by the non-linear model, it does not guarantee the convexity will still hold once the model is made discrete. Therefore, we should be careful in this transition. We will go further in these details in the next section.

The choice between primal and dual functions can be selected in accordance to the field formulation chosen, see section 3.2. The internal energy $G$ and the dissipation potential $\chi$ correspond to the 2-Field variational formulation, which is the one considered in the sequel.

\section{The discrete variational form}

This section presents the time and space discretization of the 2-Field variational formulation and a summary on the consistency between the continuum and discrete formulations. The developments shown for the 2-Field variational formulation can be extended to the 3-Field and the 1-Field variational formulations in a straightforward manner. 
5.1 Time discrete incremental variational principle

The time integral (15b) is discretized through $n$ time steps, using implicit finite difference approximation.

$$
\begin{aligned}
\Pi_{2}\left[c_{n+1}, \mu_{n+1}\right]= & \sum_{i=1}^{n}\left[\int_{\Omega}\left(\frac{\Delta G}{\Delta t}-\mu_{n+1} \frac{\Delta c}{\Delta t}-\chi\left(\boldsymbol{g}_{n+1}, c_{n+1}\right)+\mu_{n+1} f_{c}\left(t_{n+1}\right)\right) d V-\right. \\
& \left.\int_{\partial_{j} \Omega} \mu_{n+1} \bar{j}_{n+1} d S\right] \Delta t \\
= & \sum_{i=1}^{n} \Pi_{2}^{n}\left[\mu_{n+1}, c_{n+1}\right]
\end{aligned}
$$

where the $\Delta$ operator, is the time difference operator, so that $\Delta G:=G\left(c_{n+1}\right)-G\left(c_{n}\right)$, and $\Delta t:=$ $t_{n+1}-t_{n}$. This changes the problem into $n$ sequential problems, each one corresponding with one of the $n$ partitions of time. The semi-discrete functional $\Pi_{2}^{n}$ is known as the incremental variational formulation [33-35], and it is defined as

$$
\begin{aligned}
\Pi_{2}^{n}\left[\mu_{n+1}, c_{n+1}\right]:= & \int_{\Omega}\left(\Delta G-\mu_{n+1} \Delta c-\Delta t \chi\left(\boldsymbol{g}_{n+1}, c_{n+1}\right)+\Delta t \mu_{n+1} f_{c}\left(t_{n+1}\right)\right) d V \\
& -\int_{\partial_{j} \Omega} \Delta t \mu_{n+1} \bar{j}_{n+1} d S
\end{aligned}
$$

This choice is not the only way to make time discrete in a variational approach [36-39], but this is the one used here for its simplicity.

\subsection{Consistency with the continuum formulation}

The continuum variational model should be consistently represented by its discrete counterpart. Classical finite elements are used to get the set of discrete equations over the discrete domain $\Omega_{h}$. To verify consistency we check that the discrete version approaches the continuous one as temporal and spatial discretizations go to zero.

First Variations : As in the continuum case, the first variation of the functional $\Pi_{2}$ should vanish to get the discrete optimal point.

Variation with respect to $\mu_{h}$ : The variation of $\Pi_{2}^{n}$ with respect to the discrete chemical potential $\mu_{h}$ leads to

$$
<D_{\mu} \Pi_{2}^{n}, \delta \mu_{h}>\equiv 0 \quad \Rightarrow \quad \begin{cases}c_{h}=-\nabla \cdot \frac{\partial \chi}{\partial \boldsymbol{g}_{h}}+f_{c}(t) & \forall \boldsymbol{X} \in \Omega_{h} \\ \bar{j}_{h}=\left.\frac{\partial \chi}{\partial \boldsymbol{g}_{h}}\right|_{c} \cdot \boldsymbol{n} & \forall \boldsymbol{X} \in \partial_{j} \Omega_{h}\end{cases}
$$

This variation gives the discrete mass balance and the prescribed flux condition, which are consistent with their continuum counterpart.

Variation with respect to $c_{h}$ : The variation with respect to $c_{h}$ leads to

$$
<D_{\dot{c}} \Pi_{2}^{n}, \delta \dot{c}_{h}>\equiv 0 \quad \Rightarrow \quad\left\{\mu_{h}=\frac{d G}{d c_{h}}-\left.\Delta t \frac{\partial \chi}{\partial c_{h}}\right|_{g_{h}} \forall \boldsymbol{X} \in \Omega_{h}\right.
$$

A second term has been added in the right-hand-side of the discrete equation (34) with respect to its continuum counterpart (11). This will lead to an effect of the gradient of the chemical potential 
on the incremental approximation of the concentration. This is a purely algorithmic effect and is not linked to any physical phenomenon since one can always adjust the discretization by taking $c_{n+\alpha}$, $\alpha \in[0,1]$. In essence, there is always an optimal value of $\alpha$ which can provide the same result than the classical backward Euler time discretization. The optimal value of $\alpha$ will depend on the chosen material model and parameters. A similar effect involving hardening has been discussed deeper in [40]. Nonetheless, the expression (34) remains consistent when the time step tends to zero. The effect of this incremental approximation is similar to that encountered in the case of visco-plasticity, for example, as studied in [40]. One way to bring consistency back into the system, is to evaluate the concentration in the dissipation potential at time $t_{n}$ for the concentration. By doing so, the system we are solving is not a fully implicit scheme anymore, it becomes semi-implicit. The Semi-Implicit (SI) and Fully-Implicit (FI) schemes will be considered in numerical applications

$$
\begin{gathered}
\frac{\text { 2-Field discrete variational form (Semi-Implicit scheme): }}{\{c, \mu\}=\arg \inf _{c} \sup _{\mu} \Pi_{2}^{S I}[c, \mu]} \\
\Pi_{2}^{S I}[c, \mu]=\int_{\Omega_{h}}\left(\Delta G-\mu \Delta c-\Delta t \chi\left(\boldsymbol{g}, c_{n}\right)+\Delta t \mu f_{c}(t)\right) d V-\int_{\partial_{j} \Omega_{h}} \Delta t \mu \bar{j} d S
\end{gathered}
$$

The difference stems from the fact that optimization is performed in the continuum problem with respect to $\dot{c}$, while it is done with respect to $c_{n+1}$ in the discrete version. Therefore, while variations in the continuum case with respect to $\dot{c}$ and $c$ give different outputs, in the discrete form the variations with respect to $c_{n+1}$ are the only available ones due to the implicit time discretization of $\dot{c}$. On the other hand, the original formulation yields

$$
\begin{aligned}
& \frac{\text { 2-Field discrete variational form (Fully-Implicit scheme): }}{\{c, \mu\}=\arg \inf _{c} \sup _{\mu} \Pi_{2}^{F I}[c, \mu]} \\
& \Pi_{2}^{F I}[c, \mu]=\int_{\Omega_{h}}\left(\Delta G-\mu \Delta c-\Delta t \chi(\boldsymbol{g}, c)+\Delta t \mu f_{c}(t)\right) d V-\int_{\partial_{j} \Omega_{h}} \Delta t \mu \bar{j} d S
\end{aligned}
$$

\subsection{Newton solver}

The Newton method can be used for the solution of the discrete variational form. It transforms the discrete optimization problem set in (35a), (35b) or in (36a), (36b) - the semi-implicit and fullyimplicit schemes respectively - into a sequence of linear algebra problems.

Using as an example the fully implicit 2-Field variational formulation (36), the residual vector $\mathbf{R}$ and the tangent matrix $\mathbf{T}$ are constructed through the following block-matrix form

$$
\underbrace{\left[\begin{array}{ll}
\mathbb{T}_{\mu \mu} & \mathbb{T}_{\mu c} \\
\mathbb{T}_{c \mu} & \mathbb{T}_{c c}
\end{array}\right]}_{=: \mathbf{T}}\left[\begin{array}{c}
\hat{\Delta} \mu \\
\hat{\Delta} c
\end{array}\right]=-\underbrace{\left[\begin{array}{c}
\mathbb{R}_{\mu} \\
\mathbb{R}_{c}
\end{array}\right]}_{=: \mathbf{R}}
$$

where the corrections in each field are denoted by the operator $\hat{\Delta}$. Each block subindex denotes the field which we need to variate to define the block. In addition, due to the continuity in $\Pi_{2}^{F I}$ the above matrix $[\mathbf{T}]$ is symmetric (Schwarz's theorem [41]). 
Since the following solution spaces

$$
\mathcal{V}_{\mu}:=\left\{\mu \in H^{1}(\Omega) ; \mu=\bar{\mu} \forall \boldsymbol{X} \in \partial_{\mu} \Omega\right\} \quad \mathcal{V}_{c}:=\left\{c \in L^{2}(\Omega)\right\},
$$

where $L^{2}(\Omega)$ denotes square integrable functions, are consistent with the 2-Field variational form (i.e. the gradient of $\mu$ is involved while that of $c$ does not appear), the following approximations are considered:

$$
\begin{aligned}
& \mu_{h}(\boldsymbol{X}, t)=\sum_{a} N_{a}(\boldsymbol{X}) \mu^{(a)}(t) \\
& c_{h}(\boldsymbol{X}, t)=\sum_{p} \delta\left(\boldsymbol{X}_{p}-\boldsymbol{X}\right) c^{(p)}(t)
\end{aligned}
$$

where $N_{a}(\boldsymbol{X})$ denotes the finite element shape function associated with node $a, \delta$ is the Dirac delta function and $p$ stand for a Gauss integration points. Hence, the components of each block-residual and block-matrix read as follow:

$$
\begin{aligned}
\mathbb{R}_{\mu}^{(a)} & :=\frac{\partial \Pi_{2}^{F I}}{\partial \mu^{(a)}}=\int_{\Omega_{h}}\left[\begin{array}{ll}
-\Delta c & N_{\mu}^{(a)} \Delta t \frac{\partial \chi}{\partial \boldsymbol{g}} \cdot \nabla N_{\mu}^{(a)}
\end{array}\right] d V-\int_{\partial_{j} \Omega_{h}} \Delta t N_{\mu}^{(a)} \bar{j} d S \\
\mathbb{R}_{c}^{(p)} & :=\frac{\partial \Pi_{2}^{F I}}{\partial c^{(p)}}=\sum_{p}\left[\frac{d G}{d c}\left(\boldsymbol{X}_{p}\right)-\mu\left(\boldsymbol{X}_{p}\right)-\Delta t \frac{\partial \chi}{\partial c}\left(\boldsymbol{X}_{p}\right)\right] \\
\mathbb{T}_{\mu \mu}^{(a, b)} & :=\frac{\partial \mathbb{R}_{\mu}^{(a)}}{\partial \mu^{(b)}}=\int_{\Omega_{h}}-\Delta t \nabla N_{\mu}^{(a)} \cdot \frac{\partial^{2} \chi}{\partial \boldsymbol{g}^{2}} \cdot \nabla N_{\mu}^{(b)} d V \\
\mathbb{T}_{c c}^{(p, q)} & :=\sum_{p}\left[\frac{d^{2} G}{d c^{2}}\left(\boldsymbol{X}_{p}\right)-\Delta t \frac{\partial^{2} \chi}{\partial c^{2}}\left(\boldsymbol{X}_{p}\right)\right] \quad \forall q=p \\
\mathbb{T}_{\mu c}^{(a, p)} & :=\frac{\partial \mathbb{R}_{\mu}^{(a)}}{\partial c^{(p)}}=\sum_{p}\left[-N_{\mu}^{(a)}\left(\boldsymbol{X}_{p}\right)+\Delta t \nabla N_{\mu}^{(a)}\left(\boldsymbol{X}_{p}\right) \cdot \frac{\partial^{2} \chi}{\partial \boldsymbol{g} \partial c}\left(\boldsymbol{X}_{p}\right)\right] \\
\mathbb{T}_{c \mu}^{(p, a)} & :=\frac{\partial \mathbb{R}_{c}^{(p)}}{\partial \mu^{(a)}}=\sum_{p}\left[-N_{\mu}^{(a)}\left(\boldsymbol{X}_{p}\right)+\Delta t \nabla N_{\mu}^{(a)}\left(\boldsymbol{X}_{p}\right) \cdot \frac{\partial^{2} \chi}{\partial c \partial \boldsymbol{g}}\left(\boldsymbol{X}_{p}\right)\right]
\end{aligned}
$$

\section{Numerical examples}

Numerical examples in one and two space dimensions are shown here. Convergence properties and consistency between the weak and variational formulations are studied with the $1 \mathrm{D}$ results. The 2D examples we present show different results where the domain is decomposed in two different materials, both undergoing diffusion.

\subsection{Initial Dirichlet discontinuity in 1D}

A numerical example of initial-Dirichlet discontinuity is presented which proves interesting enough to solve numerically, due to the appearance of spurious oscillations even for some implicit scheme. By doing this, we show how the proposed variational formulation performs when compared with different formulations.

Initial and boundary conditions are given by the following set of equations 


\begin{tabular}{|c|c|c|}
\hline Parameter & Value & Units \\
\hline $\bar{\mu}$ & $-175.0 \cdot 10^{-3}$ & $\mathrm{~J} \mathrm{~mol}^{-1}(\mathrm{Gibbs})$ \\
\hline \hline$\mu_{0}$ & $-190.0 \cdot 10^{-3}$ & $\mathrm{~J} \mathrm{~mol}^{-1}(\mathrm{Gibbs})$ \\
\hline$c_{0}$ & $\mathrm{Eq}(21)$ or Eq $(2 \mathrm{~b})$ & $\mathrm{mol} \mathrm{m}^{-3}$ \\
\hline \hline$R$ & 8.3144598 & $\mathrm{~kg} \mathrm{~m}^{2} \mathrm{sec}^{-2} \mathrm{~K}^{-1} \mathrm{~mol}^{-1}$ \\
\hline$T$ & 298.0 & $\mathrm{~K}$ \\
\hline$D$ & $0.84 \cdot 10^{-9}$ & $\mathrm{~m}^{2} \mathrm{sec}^{-1}$ \\
\hline \hline$L$ & $1.0 \cdot 10^{-3}$ & $\mathrm{~m}$ \\
\hline$t_{f}$ & 1190.47619048 & $\mathrm{sec}$ \\
\hline
\end{tabular}

Table 1: Table of parameters

*The values for $c_{0}$ are made through the models from the given equations.

\section{Boundary conditions}

$$
\begin{aligned}
& j(0, t)=0 \\
& c(L, t)=c_{\text {ext }}
\end{aligned}
$$

\section{$\underline{\text { Initial conditions }}$}

$$
\begin{aligned}
& \left.c(x, 0)=c_{0} \quad \forall x \in\right] 0, L[ \\
& \text { with } c_{0} \neq c_{\text {ext }}
\end{aligned}
$$

with the spatial domain defined as $\Omega:=] 0, L\left[\right.$ and temporal domain $\tau:=\left[0, t_{f}\right]$, with $t_{f}:=L^{2} / D$. The boundary condition (41a) at the left of $\Omega$ imposes a zero flux condition. The condition (41b) sets a Dirichlet value for the concentration at the right. Finally the initial condition (42) is defined as constant and imposes a discontinuity between the Dirichlet and the initial values.

The analytical solution of this problem is found in [42]. Two results are presented. First, a solution based on trigonometric functions that converge rapidly for large values of time $t$. See [42, p. 24].

$$
\frac{c(x, t)-c_{0}}{c_{e x t}-c_{0}}=\left(1-\frac{4}{\pi} \sum_{m=0}^{\infty} \frac{(-1)^{m}}{2 m+1} \exp \left(-D\left[\frac{(2 m+1) \pi}{2 L}\right]^{2} t\right) \cos \frac{(2 m+1) \pi x}{2 L}\right)
$$

The second one is expressed through a series of complementary error functions, which converge rapidly for all time values except large ones [42, p. 22]

$$
\frac{c(x, t)-c_{0}}{c_{e x t}-c_{0}}=\sum_{m=0}^{\infty}(-1)^{m}\left(\operatorname{erfc} \frac{(2 m+1) L-x}{2 \sqrt{D t}}+\operatorname{erfc} \frac{(2 m+1) L+x}{2 \sqrt{D t}}\right)
$$

Figure 1 depicts the analytical solution, computed with the values listed in table 1 . A plane cutting the space and time domains at their half is presented in each subfigure for visualization purpose. The plane in figure 1a describes the evolution of the concentration at all times for $x=L / 2$, while that in figure $1 \mathrm{~b}$ can be seen as a snapshot of the space domain at time $t=t_{f} / 2$.

\subsubsection{Classical weak form based numerical solution}

The matrix and the residual vector are defined as

$$
\begin{aligned}
\mathbb{A}^{(a, b)} & :=\int_{\Omega_{h}} N^{(a)} N^{(b)} d x+\int_{\Omega_{h}} D \Delta t \nu \nabla N^{(a)} \cdot \nabla N^{(b)} d x \\
\mathbb{R}^{(a)} & :=c_{n}^{(a)}\left[\int_{\Omega_{h}} N^{(a)} N^{(b)} d x-\int_{\Omega_{h}}\left(D \Delta t(1-\nu) \nabla N^{(a)} \cdot \nabla N^{(b)}\right) d x\right] \\
& +\int_{\Omega_{h}} \Delta t r_{n+\nu} d x
\end{aligned}
$$




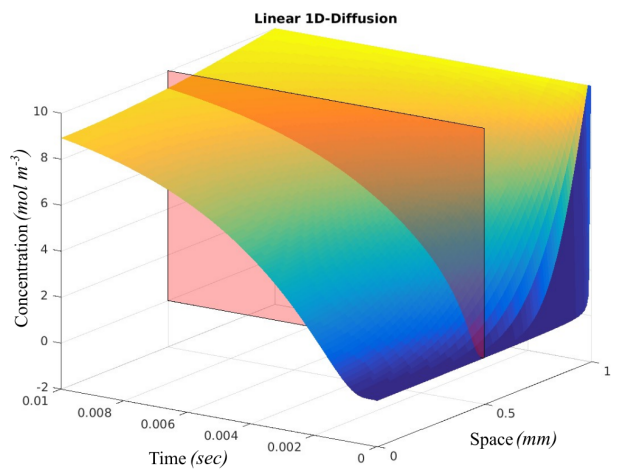

(a) Cross section: time evolution of the solution at the location $x=L / 2$.

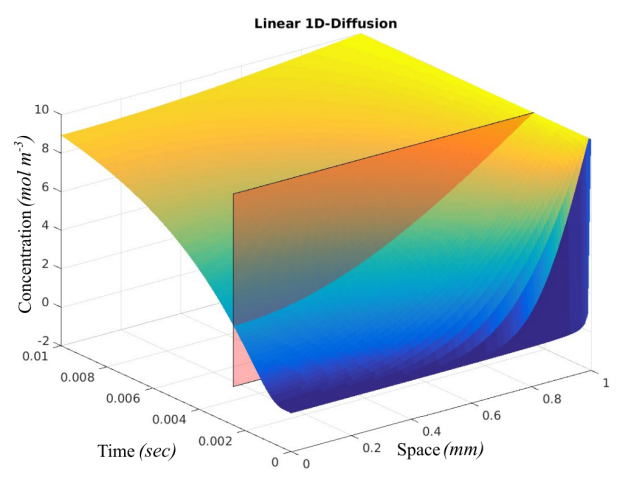

(b) Cross section: snapshot of the solution at time $t=t_{f} / 2$.

Fig. 1: Analytical solution: plot of the concentration as a function of $x$ and $t$.

where $\nu \in[0,1]$ is the algorithm parameter. Letting $\nu \equiv 0$ gives an explicit scheme, while $\nu \equiv 1$ turns the system of equations into the Euler implicit scheme. There are two types of spurious oscillations that may arise in this problem imposing a lower and/or upper bound to the time step. A stability analysis of the system shows an upper limit condition $\Delta t_{\text {max }}$ for the discrete time step $\Delta t .^{\S} \mathrm{A}$ second type of spurious oscillations rises as a constraint needed in order to satisfy the discrete maximum principle in Fick's second law (1) [43, p. 126]. The critical time step is given by

$$
\Delta t>\Delta t_{\min } \quad \text { where } \quad \Delta t_{\min }:=\frac{h_{\min }^{2}}{6 D \nu}
$$

where $h_{\min }$ is the size of the smallest element.

\subsubsection{Implicit scheme vs Semi-implicit schemes}

To perform the analysis, we consider the parameters listed in table 1 along with $\nu=1$ and $N_{x}=512$ spatial elements. We consider $N_{t}$ equally spaced time steps over a simulation time defined as the

\footnotetext{
$\S_{\text {see }}[43$, p. 124] for an example of an analytical approximation.
} 
diffusion characteristic time computed with the length of the domain $t_{f}=L^{2} / D$. Thus, letting $\Delta t \equiv \Delta t_{\min }$ defines the threshold to obtain the lower boundary for a spurious oscillation

$$
N_{t_{o s c}}:=\frac{t_{f}}{\Delta t_{\min }}=\frac{L^{2} / D}{\left(L / N_{x}\right)^{2} /(6 D)}=6 N_{x}^{2}
$$

hence $N_{t}>6 N_{x}^{2}$ ensures spurious oscillations will appear with linear elements. The collection of time steps $N_{t_{\text {list }}}$ we use to define the tests is $N_{t_{\text {list }}}:=\left\{2^{3}, 2^{4}, \ldots, 2^{13}\right\}$.

The results are presented in figures 2, 3, 4 and 5. Each one is composed of two subfigures. The one at the top represents the results where the number of spatial elements is fixed with $N_{x}=512$ for all simulations, while the number of temporal steps in each run is selected from $N_{t} \in N_{t_{l i s t}}$, we reference this process as Test 1 . The subfigures at the bottom show the results where the ratio $N_{x} / N_{t}$ is equal for all runs, while $N_{t}$ is chosen from $N_{t} \in N_{t_{l i s t}}$ and $N_{x}$ is selected accordingly, we call this simulation Test 2. In specific, we have chosen the ratio $N_{x} / N_{t}=1$ to ensure we are above the limit $\Delta t_{\text {min }}$ independently of the number of elements $N_{x}$ selected according to (46). Each subfigure contains two subplots to show the errors for the concentration (left) and chemical potential (right). In all subplots, the horizontal and vertical axis show the time step (seconds) and a certain relative error norm. The numbers between each segment of the curves represent the slope at that segment. All the errors are computed with respect to the analytical solution.

To identify each run we use the following label system: SCHEME + Model + ELEMENT TYPE which are defined as

$$
\begin{aligned}
& \text { SCHEME }:=\{\text { Fully Implicit }(\mathrm{FI}), \text { Semi-Implicit }(\mathrm{SI})\} \\
& \text { Model }:=\{\text { Linear, Fickean }\} \\
& \text { ELEMENT TYPE }:=\{\text { linear(ele_lin), quadratic(ele_quad) }\}
\end{aligned}
$$

$L^{2}$ relative error at time $t_{f}$ : Figures 2 and 3 show convergence curves for the Linear and Fickean model respectively, using the $L^{2}$ relative error at time $t_{f}$. Figures $2 \mathrm{a}$ and $3 \mathrm{a}$ show the results for the simulations under the Test 1 , while figures $2 \mathrm{~b}$ and $3 \mathrm{~b}$ correspond to the Test $2\left(\right.$ with $\left.N_{x} / N_{t}=1\right)$.

Figure 2 shows that for the Linear model, the error of the chemical potential is one order of magnitude less than that of the concentration, fact which can not be achieved using a post-process relation. However, both graphs have a convergence rate of one as expected. As it is for the Fickean model in figure 3, we also have a convergence rate of one, however, a distinction between semi-implicit and fully-implicit is observed in the Fickean model. The samples taken for the fully-implicit scheme have a lower y-intercept on the error. Still, the main difference resides on the scheme used, the type of element seems not to play a major role. A difference in the order of the error between chemical potential and concentration can also be observed, but now, the difference in the order of magnitude is two. A final comparison between errors in the Linear and Fickean model shows that the error for the chemical potential is the same despite the problem being linear or non-linear, in contrast with the concentration where the order of magnitude increases by an order of one for the non-linear (Fickean) model.

Mean $L^{1}$ relative error per time step: Figures 4 and 5 show the convergence for the Linear and Fickean model correspondingly, using the $L^{1}$ relative error per time step. For the Linear model, fully-implicit or semi-implicit schemes yield the same result, the same holds for the type of element 

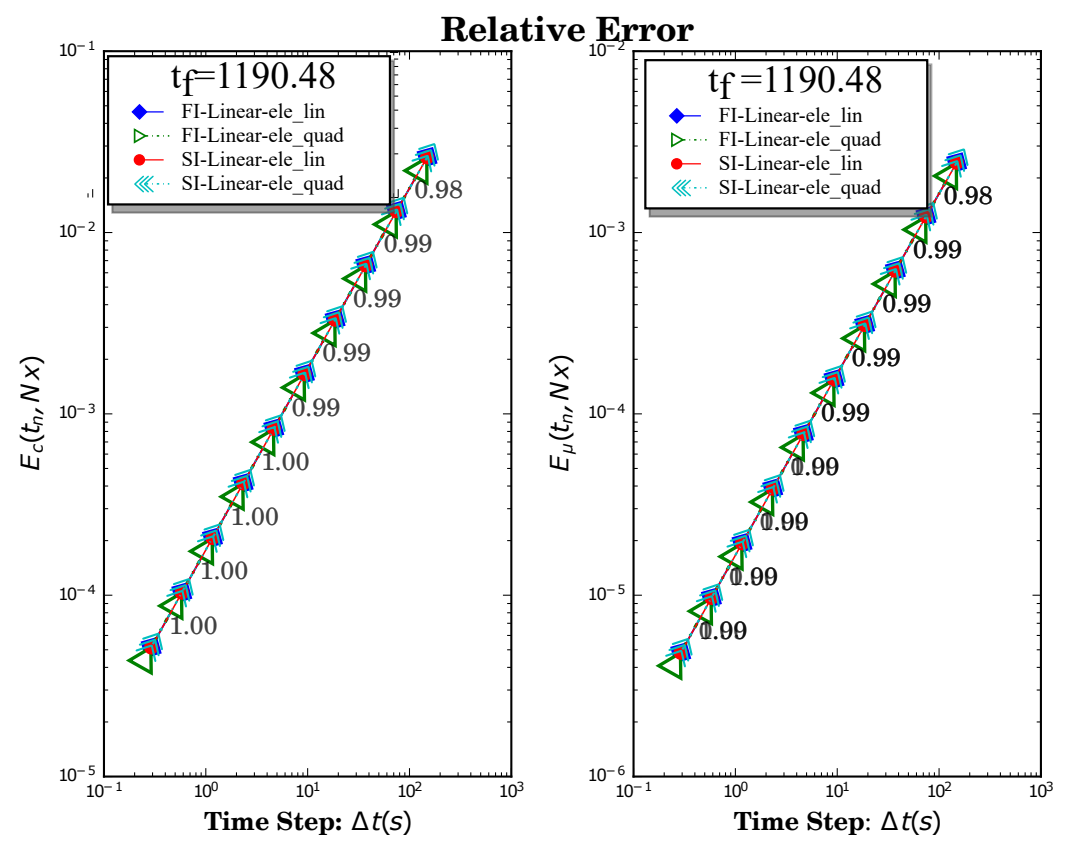

(a) Test 1: $N_{x}=512, N_{t} \in N_{t_{l i s t}}$

Relative Error
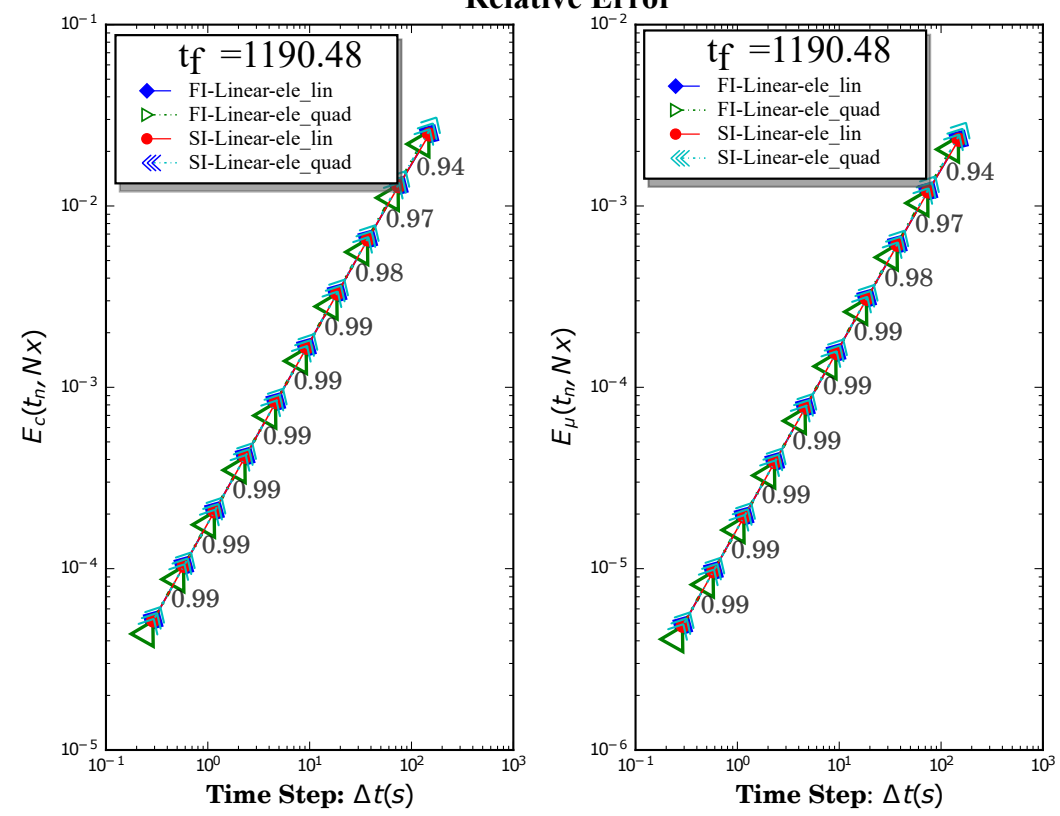

(b) Test $2: N_{x} / N_{t}=1$

Fig. 2: Convergence curves plotted for the Linear model, with the $L^{2}$ relative error computed at time $t_{f}$, and $N t_{\text {list }}=\left\{2^{3}, 2^{4}, \ldots, 2^{13}\right\}$ 

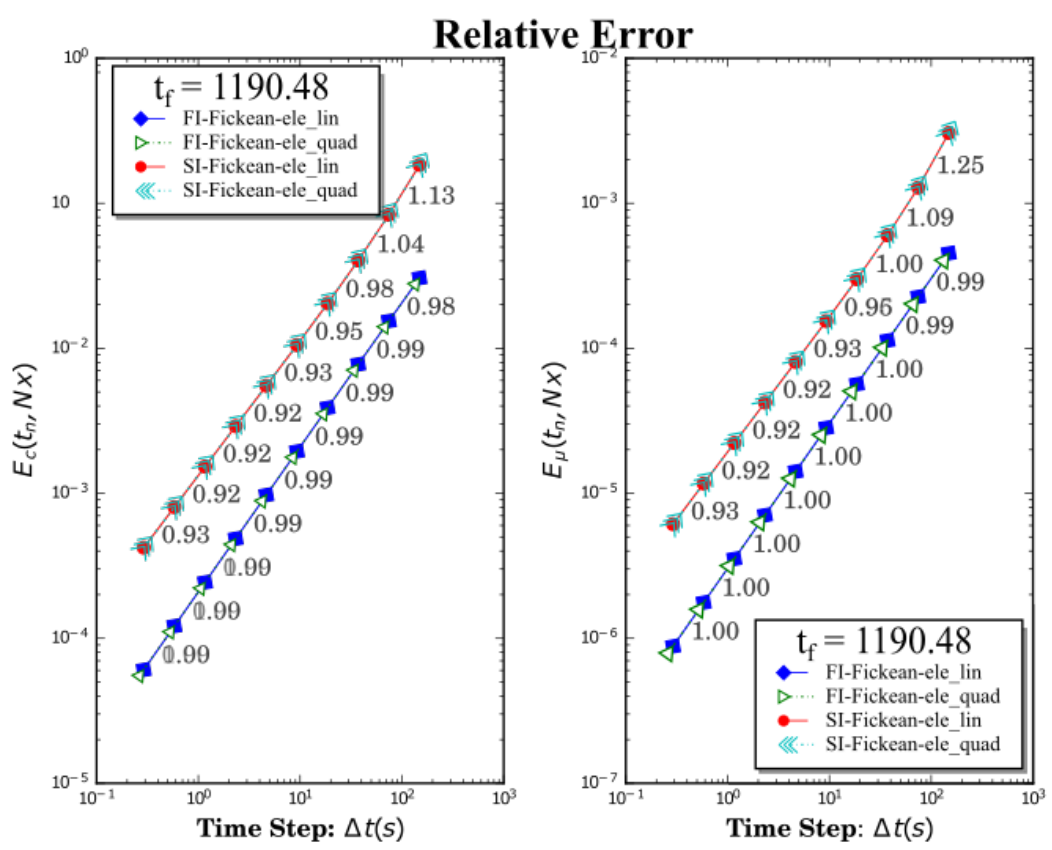

(a) Test 1: $N_{x}=512, N_{t} \in N_{t_{l i s t}}$
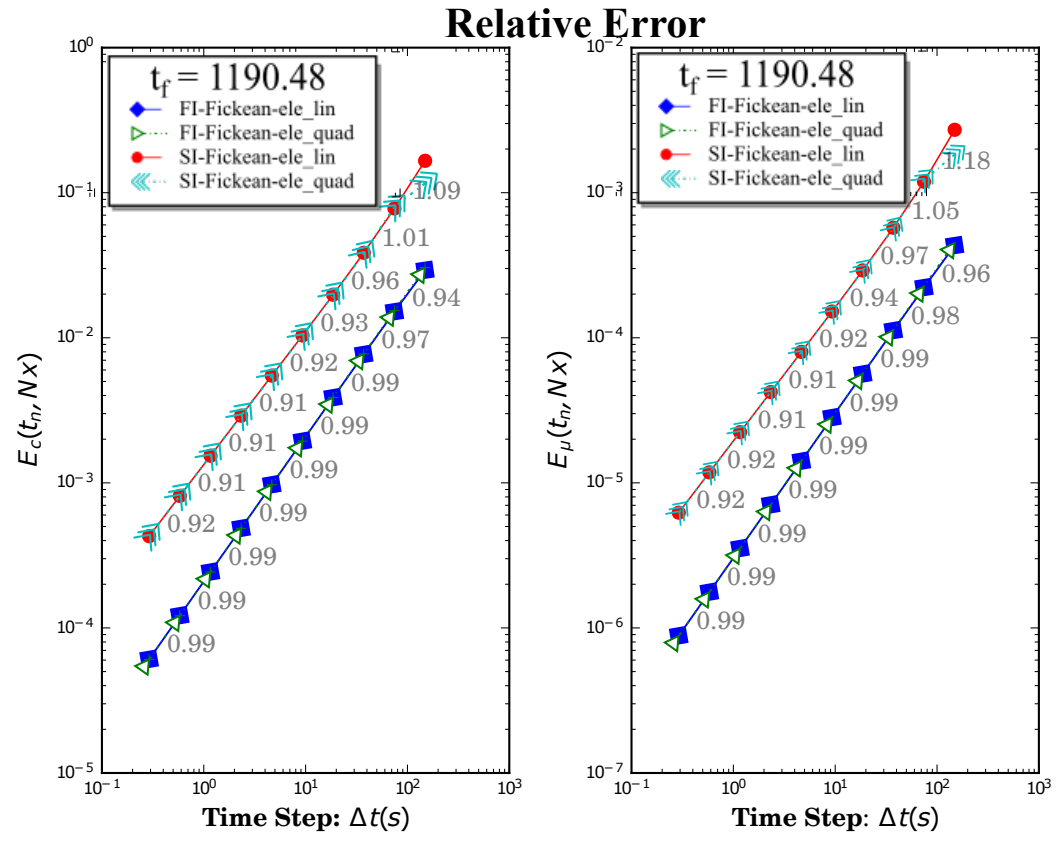

(b) Test 2: $N_{x} / N_{t}=1$

Fig. 3: Convergence curves plotted for the Fickean model, with the $L^{2}$ relative error computed at time $t_{f}$, and $N t_{\text {list }}=\left\{2^{3}, 2^{4}, \ldots, 2^{13}\right\}$ 
- linear or quadratic-, or the magnitude of the errors. The Fickean model shows the same differences between schemes and types of elements in comparison with the relative error at fixed time. Nevertheless, here the difference of the magnitude of the errors between the concentration and the chemical potential only differs by one. As shown the pairs (Figure 4a, Figure 4b) and (Figure 5a, Figure 5b) results show similar results. Therefore, the spatial and temporal discretization seem to not incorporate further artifacts to the solution besides the aforementioned spurious oscillations.

\subsubsection{Weak Form vs Variational Form}

Here we compare the fully implicit variational formulation against the weak formulation of the problem presented in (6). The discretization of the weak formulation has been also implemented using the backward Euler method for time and classic finite element method for space. The results for the Linear model and the Fickean model show congruent results. The result obtained from the Fickean model are shown in figure 6 . Both formulations show a similar convergence behavior for each model. The results for the Fickean model shown in Figure 6 show convergence rate of one as expected, achieved once the time step is sufficiently fine.

\subsection{Diffusion in a 2D composite medium}

Let's consider a 2D square domain which consists of several circular inclusions of different radii embedded in an arbitrary manner into a matrix. The two components follow the non-linear Fickean model (see §4.4), but have different material properties, whose values are listed in Table 2 . The material properties of the matrix and the inclusions are set so that the diffusion is faster in the matrix than in the inclusions. Besides, the simulations presented in this section have been performed using both the 2-Field Variational formulation (15) and the weak formulation (see §2.2), and run with the in-house code ZorgLib developed at GeM-Ecole Centrale de Nantes. Next, two different loading conditions are successively considered below, and allow to compare results obtained with both formulations.

\begin{tabular}{|c|c|c|}
\hline Parameter & Value & Units \\
\hline $\bar{\mu}$ & $-181.0 \cdot 10^{3}$ & $\mathrm{~J} \mathrm{~mol}^{-1}(\mathrm{Gibbs})$ \\
\hline $\bar{c}$ & 1000 & $\mathrm{~mol} \mathrm{~m}$ \\
\hline$\hat{\mu}_{0}$ & $-5.52 \cdot 10^{-3}$ & - \\
\hline$c_{0}$ & $\mathrm{Eq}(21)$ or Eq $(2 \mathrm{~b})$ & $\mathrm{mol} \mathrm{m}^{-3}$ \\
\hline$\mu_{0}$ & $-181.0 \cdot 10^{3}$ & $\mathrm{~J} \mathrm{~mol}^{-1}(\mathrm{Gibbs})$ \\
\hline \hline$R$ & 8.3144598 & $\mathrm{~kg} \mathrm{~m}^{2} \mathrm{sec}^{-2} \mathrm{~K}^{-1} \mathrm{~mol}^{-1}$ \\
\hline$T$ & 298.0 & $\mathrm{~K}$ \\
\hline$D_{\text {matrix }}$ & $5.0 \cdot 10^{-10}$ & $\mathrm{~m}^{2} \mathrm{sec}^{-1}$ \\
\hline$D_{\text {inclusion }}$ & $5.0 \cdot 10^{-13}$ & $\mathrm{~m}^{2} \mathrm{sec}^{-1}$ \\
\hline \hline$\Delta t$ & 5.0 & $\mathrm{~seconds}^{2}$ \\
\hline
\end{tabular}

Table 2: Table of parameters for the 2D diffusion test case.

\subsubsection{Sine loading}

Dirichlet boundary conditions are prescribed on the whole boundary of the square domain: the chemical potential is prescribed on the boundary in the 2-Field Variational formulation, and follows a sine 

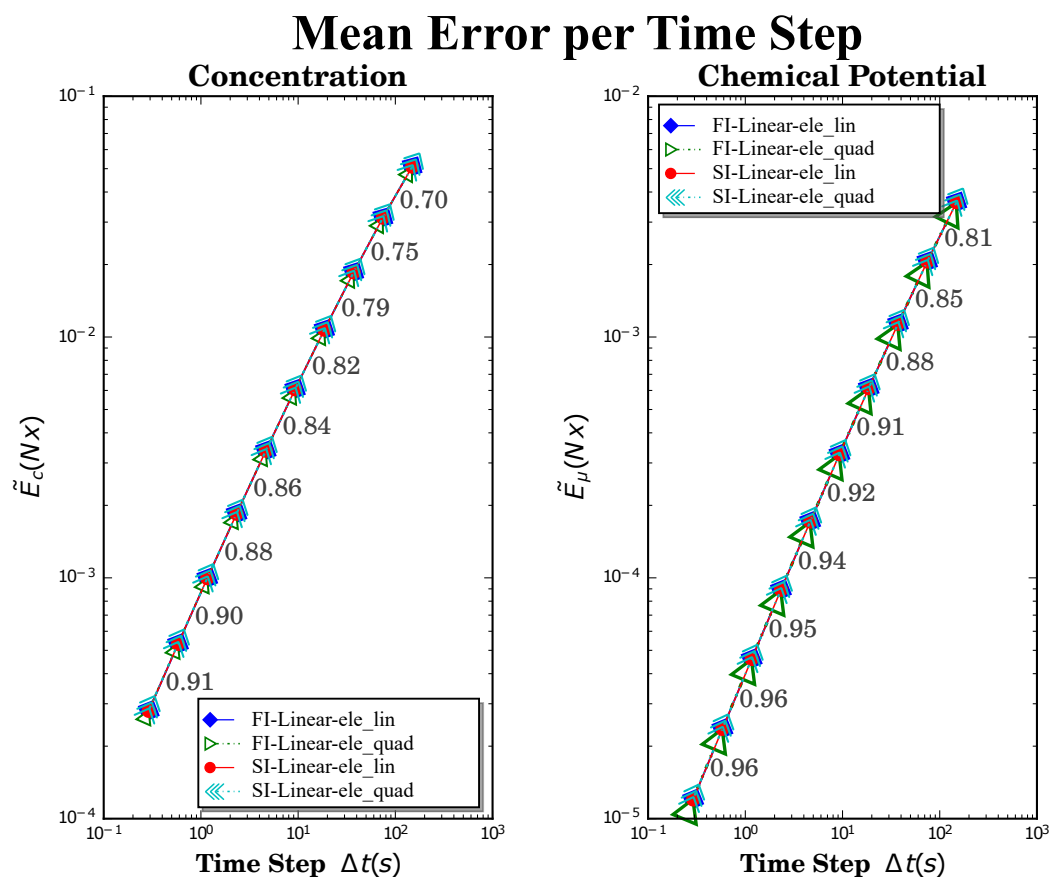

(a) Test 1: $N_{x}=512, N_{t} \in N_{t_{l i s t}}$
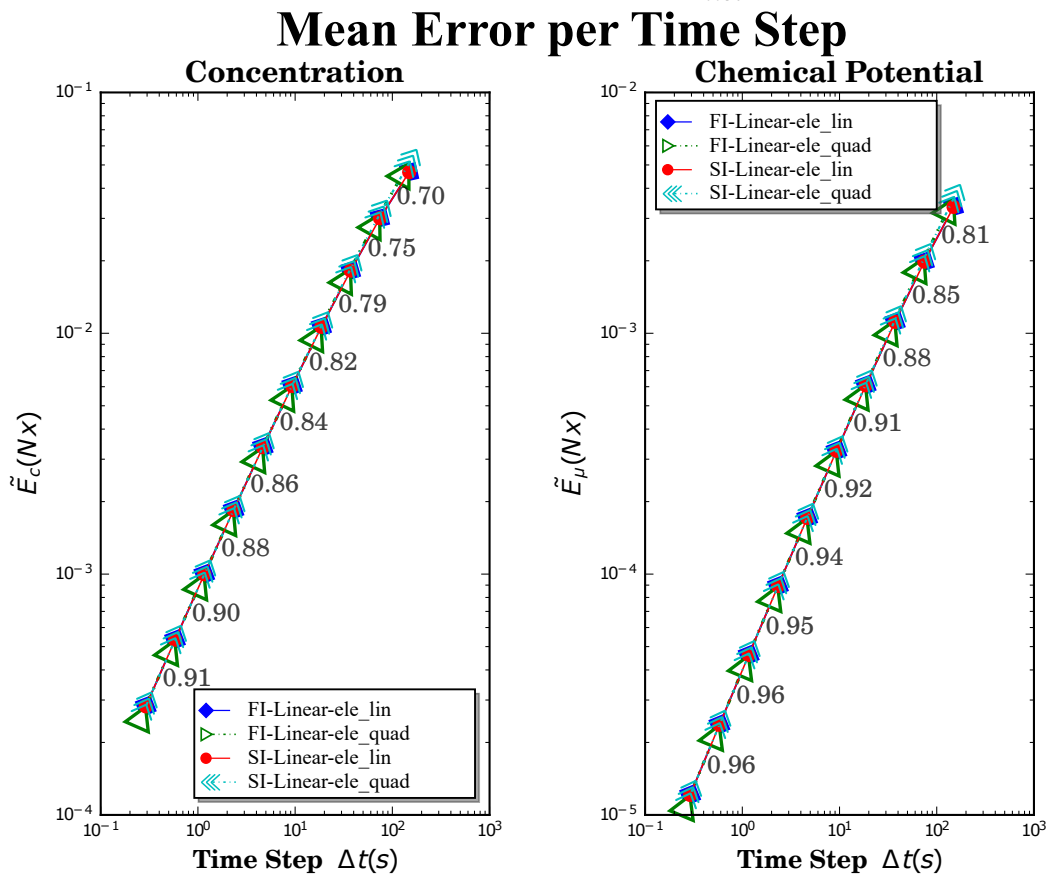

(b) Test 2: $N_{x} / N_{t}=1$

Fig. 4: Convergence curves plotted for the Linear model, with the mean $L^{1}$ relative error per time step, and $N t_{\text {list }}=\left\{2^{3}, 2^{4}, \ldots, 2^{13}\right\}$ 


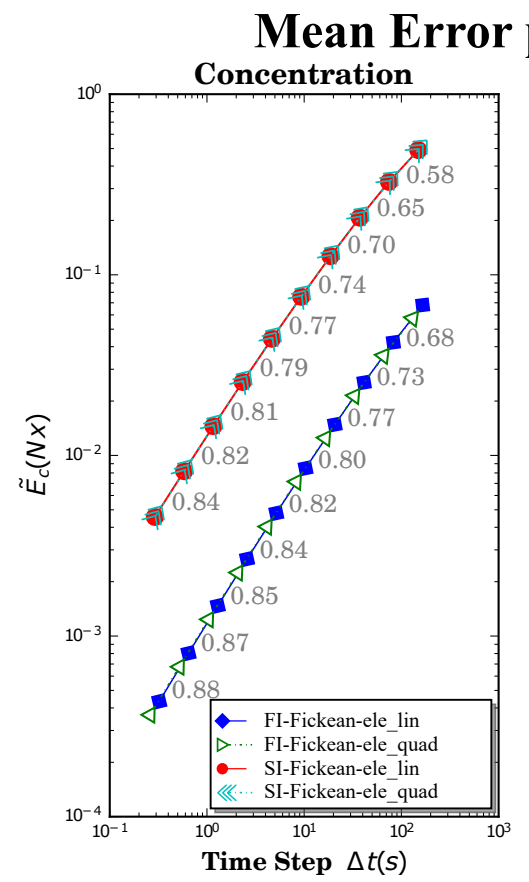

\section{per Time Step}

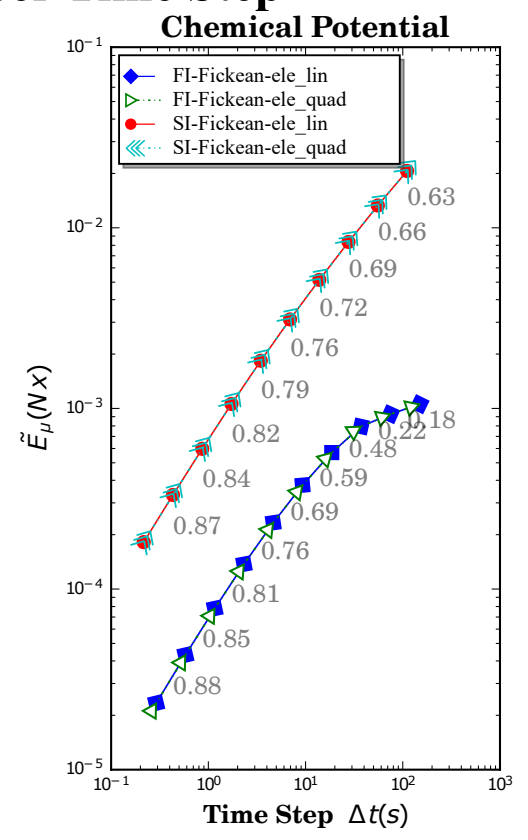

(a) Test 1: $N_{x}=512, N_{t} \in N_{t_{l i s t}}$
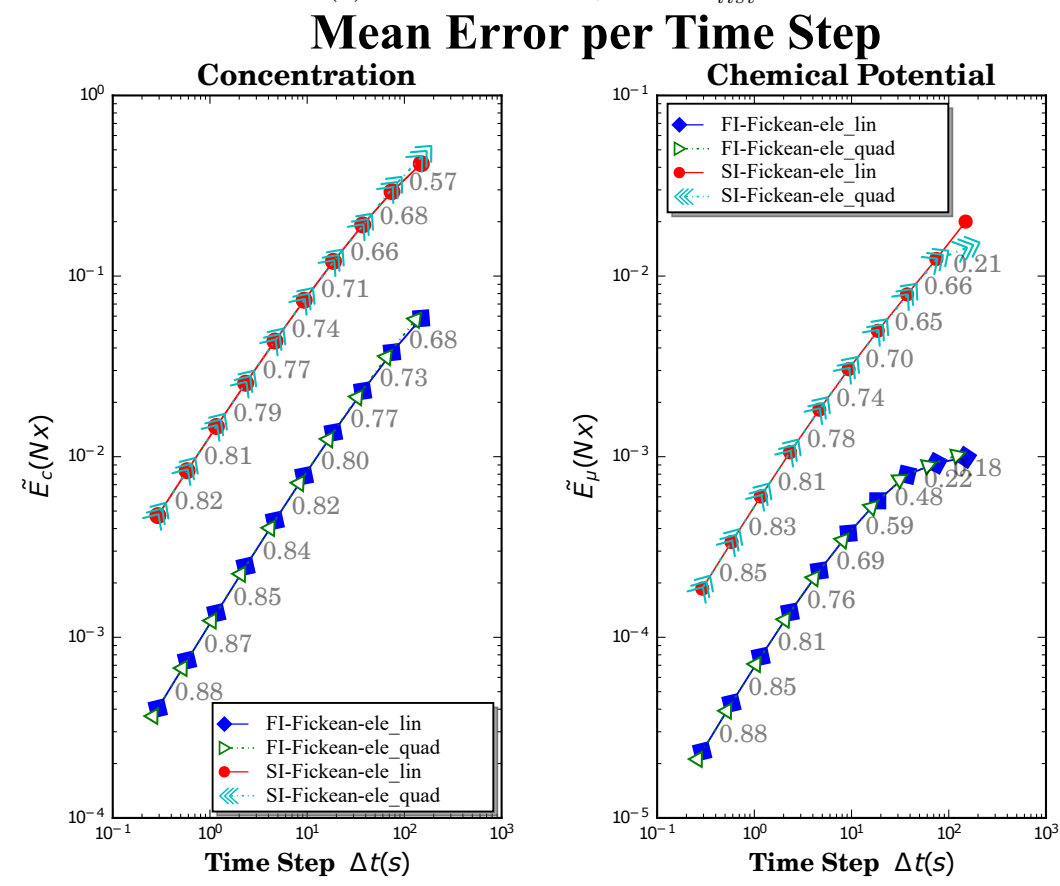

(b) Test 2: $N_{x} / N_{t}=1$

Fig. 5: Convergence curves plotted for the Fickean model, with the mean $L^{1}$ relative error per time step, and $N t_{\text {list }}=\left\{2^{3}, 2^{4}, \ldots, 2^{13}\right\}$ 

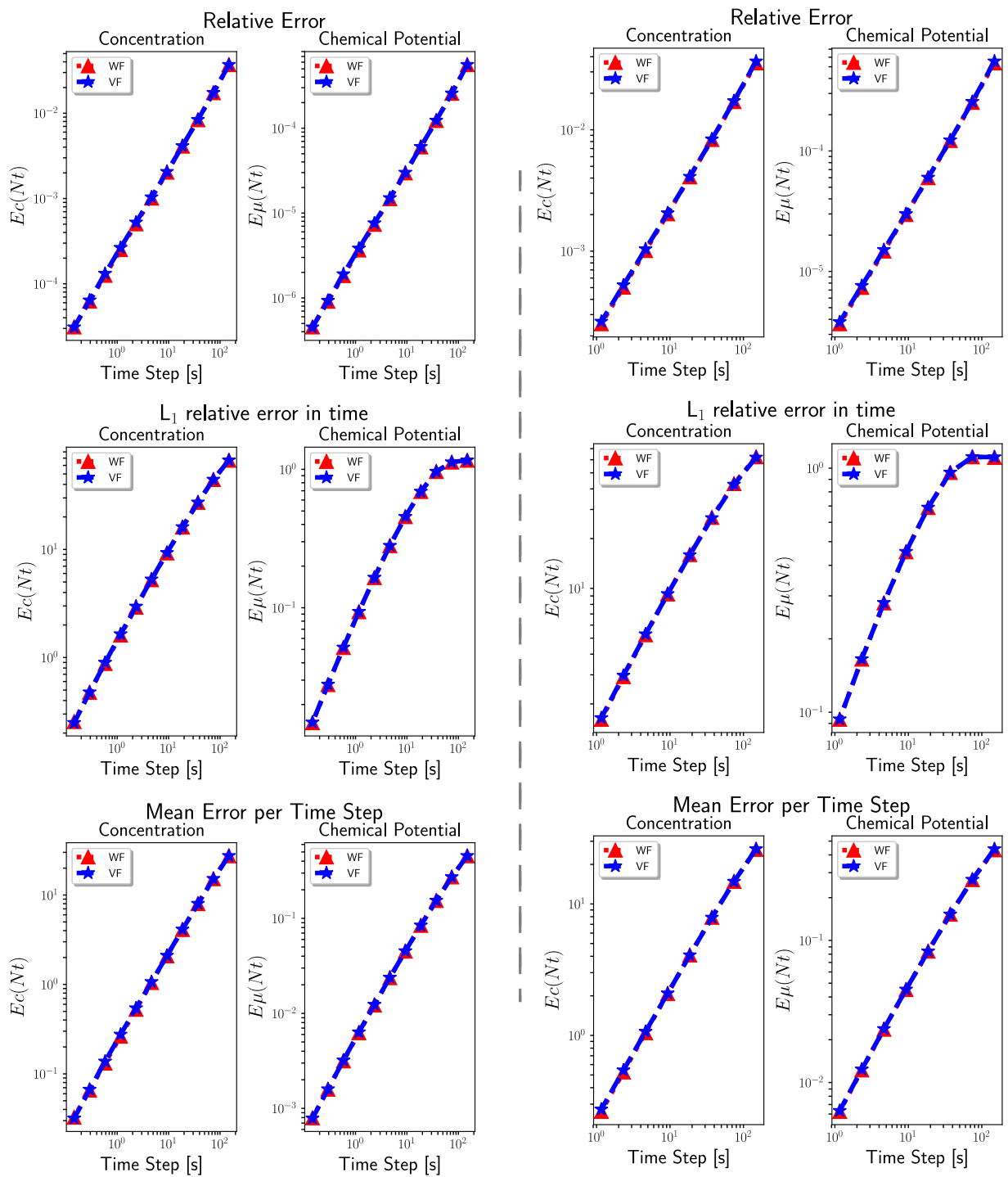

Fig. 6: Comparison between convergence curves obtained with the weak and the variational forms, plotted for the Fickean model. Three types of error are plotted: $L^{2}$ relative error computed at time $t_{f}$ (first row), $L^{1}$ relative error in time (second row), and the mean $L^{1}$ relative error per time step (third row). Plots associated with Test 1 are shown on the left, while these associated with Test 2 are shown on the right.

evolution in time:

$$
\mu_{\text {prescribed }}(t)=\bar{\mu} f(t), \quad f(t)=1+\hat{\mu}_{0} \sin \left(\frac{2 \pi t}{P}\right)
$$

where $\hat{\mu}_{0}$ is a dimensionless parameter, $P=250$ seconds is the loading period. The concentration defined at boundary nodes in the case of the weak formulation is computed from the prescribed value of the chemical potential using the mass action law (2b). Some snapshots of the chemical 
potential, extracted from one period of loading, are shown at different times in Figure 7a and 7b for the variational and weak formulations respectively. The chemical potential is shown as nodal values for both formulations, as a direct result from the discrete set of equations (40a) for the variational formulation, and by postprocessing the concentration nodal values with the mass action law (2b) for the weak formulation.

As expected, the inclusions diffuse slower than the matrix. A good agreement between the two sets of solution can be observed at the different times, althought slight differences between both appear at large times. The differences can be attributed to the different approximations performed in the respective formulations.

\subsubsection{One-dimensional mass flow: filling of a cavity}

The composite computational domain is now considered as an insulated cavity, which is filled through a given mass flow. The top, the bottom and the right sides of the computational domain are thus prescribed a zero mass flux, while a given influx $\bar{j}$ is prescribed on its left side. The time evolution of the influx $\bar{j}$ follows a ramp from the initial value $-181.0 \cdot 10^{3} \mathrm{~J} \mathrm{~mol}^{-1}$ to $-180.0 \cdot 10^{3} \mathrm{~J} \mathrm{~mol}^{-1} \mathrm{during}$ the first 200 seconds, after what it remains constant. Figure $8 \mathrm{a}$ and $8 \mathrm{~b}$ show some snapshots of the chemical potential field at different times, obtained with the variational and the weak formulations respectively. The presented snapshots cover a wide time range starting from the homogeneous initial condition and a very early instant of diffusion to a time where the matrix has almost reached a steadystate while inclusions still remain transient. Again, a very good agreement is observed between results obtained with both formulations, especially in inclusions where the highest gradients occur.

\section{Conclusions}

In this article, we have proposed an approach to build variational principles for the diffusion Initial Boundary Value problem. This approach has several advantages. The variational principles embed the mass action law and Fick's laws leading to a model where all the unknown fields are implicitly dependent. This contrasts with usual approaches where the chemical potential and the mass flux depend explicitly on the concentration. Second, the initially derived variational principle allows to identify the chemical potential as the opposite of the Lagrange multiplier that allows to enforce the balance of mass and the natural boundary condition. Third, the relation between the mass flux and the chemical field as conjugate variables is derived from it. Fourth, it allows to construct three variational formulations described by one, two or three fields. Fifth, it allows a flexible framework to represent constitutive models which are thermodynamically consistent. The Linear and Fickean model have been presented. Sixth, the approach is based on general concepts as the Generalized Standard Material framework, balance laws, constrained optimization and thermodynamic variables.

As part of the results, we analyzed and checked the consistency of the proposed variational formulations with the strong form of the problem. In equation (15), we arrived at the variational formulation derived by [1]. However, the same result was reached by proposing a constrained variational problem and a field reduction, while [1] started from the strong form of the problem, identified the first variation of some functional using a variational derivative, and deduce its final form through integration. In addition, we presented and analyzed the numerical results of the 1D initial Dirichlet discontinuity problem using the 2-Field variational formulation. The numerical results obtained from the $1 \mathrm{D}$ and 2D simulations confirmed that the implementation of the variational formulation shows the expected results in comparison with the weak formulation of the problem, regardless of the mass action law used for the chemical potential. However, we recall the variational formulation solves for concentration 

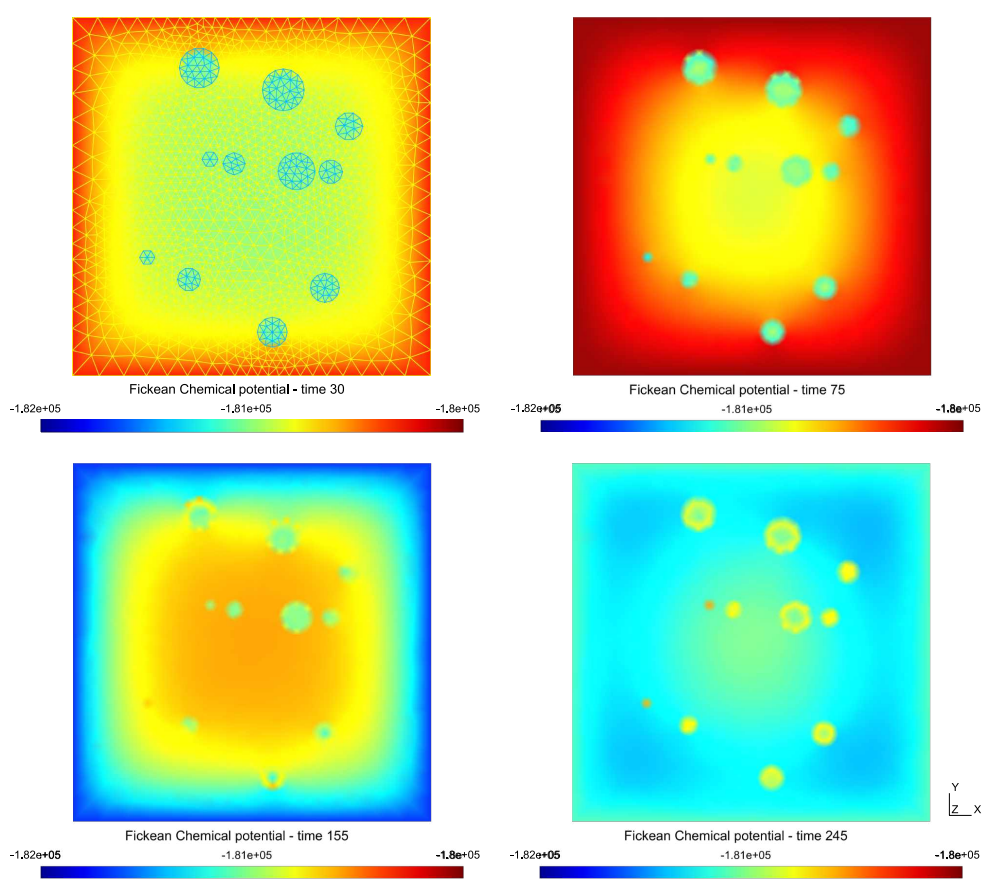

(a) Variational formulation

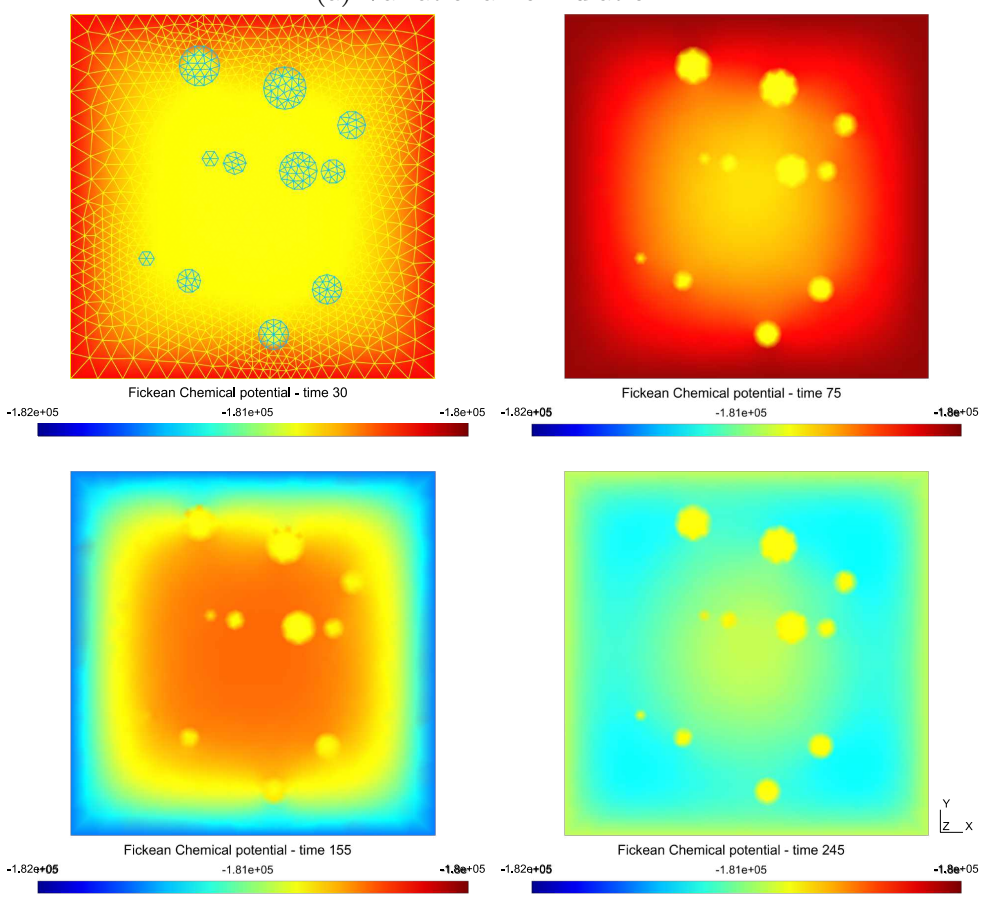

(b) Weak formulation.

Fig. 7: Sine loading 

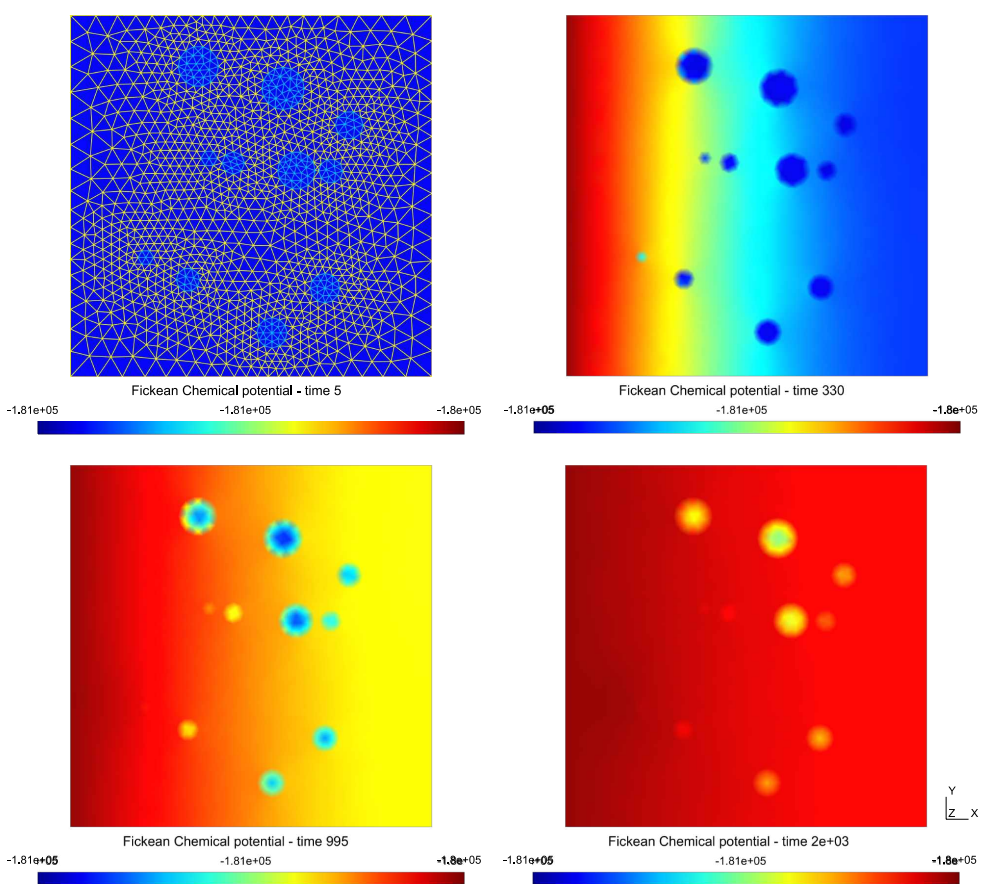

(a) Variational formulation.
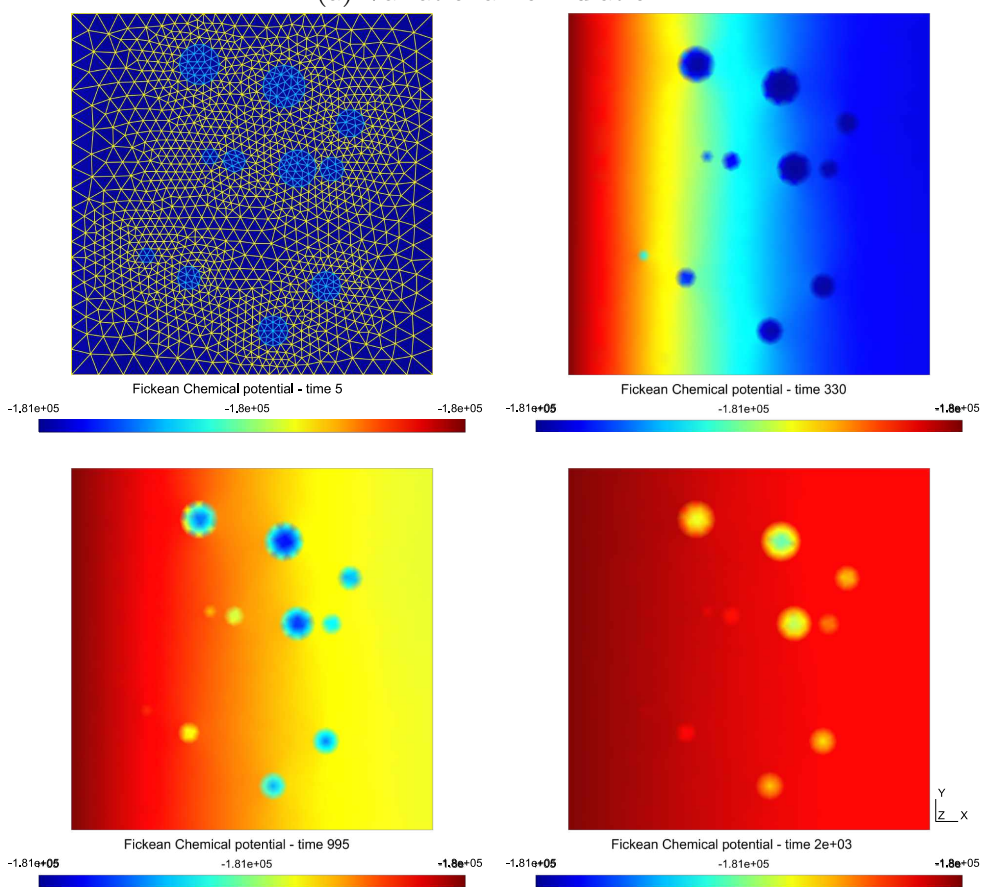

(b) Weak formulation.

Fig. 8: One-dimensional mass flow 
and chemical potential simultaneously, which contrasts with the post-process step taken to compute the chemical potential using a weak formulation. Therefore, the variational formulation provides a more robust model suitable for multiphysic problems, where the interaction of the chemical potential with other force-like fields needs to be considered simultaneously for the whole system.

From the computational perspective, it is possible to derive different -not necessarily monolithicnumerical strategies which have a mathematical representation and are consistent with the continuum model. These strategies can be derived by imposing different algorithmic dependencies between the unknowns. This is a viable option, since boundary, constitutive and balance equations are embedded in the functional and potentials of the proposed variational principle.

Finally, the proposed approach can be generalized to multiphysic problems. Constraining Onsager's energy rate with a balance law and prescribed flux-like field at the boundary is a mathematical procedure that enforces these equations into the solution and allows to define energetically-conjugate variables through the postulated internal energy and dissipation potential. Moreover, the involved fields can be easily identified as flux-like, force-like or displacement-like fields. The multiphysic coupling can be imposed under the definition of the internal energy and dissipation potential through the linear and bilinear addition of the respective fields. As an instance, for an electrochemical problem the coupling occurs between mass flux and electric current [44]. These variables are defined in terms of the chemical and the electrical field. Using our approach, the mass flux and the chemical field are energetically-conjugate and a similar relation can be derived for the electric current and the electric potential. The advantage of the former is that it allows to construct a dissipation potential to impose the coupling and at the same time to build a variational principle through the procedure proposed in this work.

Acknowledgements Jorge de Anda Salazar was funded by EMJD-SEED program, financed by the European Commission with grant Ref. 2013-0043

\section{Conflict of interest}

The authors declare that they have no conflict of interest.

\section{References}

1. C. Miehe, S. Mauthe, H. Ulmer, International Journal for Numerical Methods in Engineering 99(10), 737 (2014). DOI $10.1002 /$ nme.4700

2. A. Fick, Philosophical Magazine Series 4 10(63), 30 (1855). DOI 10.1080/14786445508641925

3. G. Job, R. Rüffler, R. Fuchs, H. Fuchs, Physical Chemistry from a Different Angle: Introducing Chemical Equilibrium, Kinetics and Electrochemistry by Numerous Experiments (Springer International Publishing, 2015)

4. J.W. Gibbs, American Journal of Science s3-16(96), 441 (1878). DOI 10.2475/ajs.s3-16.96.441

5. G. Cook, R.H. Dickerson, American Journal of Physics 63(8), 737 (1995). DOI 10.1119/1.17844

6. R. Baierlein, American Journal of Physics 69(4), 423 (2001). DOI 10.1119/1.1336839

7. G. Job, F. Herrmann, European Journal of Physics 27(2), 353 (2006)

8. L. Onsager, Phys. Rev. 37, 405 (1931). DOI 10.1103/PhysRev.37.405

9. L. Onsager, Annals of the New York Academy of Sciences 46, 241 (1945)

10. J. Svoboda, I. Turek, F.D. Fischer, Philosophical Magazine 85(31), 3699 (2005). DOI 10.1080/14786430500267760

11. K. Hackl, F.D. Fischer, J. Svoboda, Proceedings of the Royal Society A: Mathematical, Physical and Engineering Sciences 467(2128), 1186 (2010). DOI 10.1098/rspa.2010.0179

12. H.B. Callen, Thermodynamics and an Introduction to Thermostatistics (Wiley, 1985)

13. R.C. Balescu, Equilibrium and Non-Equilibrium Statistical Mechanics (Wiley, 1975) 
14. H. Ziegler, An Introduction to Thermomechanics (NORTH-HOLLAND SERIES IN APPLIED MATHEMATICS AND MECHANICS) (Elsevier Science Ltd, 1983)

15. J. Merker, M. Krüger, Continuum Mechanics and Thermodynamics 25(6), 779 (2013)

16. G. Romano, M. Diaco, R. Barretta, Continuum Mechanics and Thermodynamics 22(3), 177 (2010)

17. B. Halphen, Q. Nguyen, J Mec 14, 39 (1975)

18. M.A. Biot, Journal of Applied Physics 27(3), 240 (1956). DOI 10.1063/1.1722351

19. U. Fischer, ZAMM - Journal of Applied Mathematics and Mechanics / Zeitschrift für Angewandte Mathematik und Mechanik 64(1), 70 (1984). DOI 10.1002/zamm.19840640121. URL https://doi.org/10.1002/zamm.19840640121

20. E. Reissner, Journal of Mathematics and Physics 29(1-4), 90 (1950). DOI 10.1002/sapm195029190. URL https://doi.org/10.1002/sapm195029190

21. C.A. Felippa, Communications in Applied Numerical Methods 5(2), 79 (1989). DOI 10.1002/cnm.1630050204. URL https://doi.org/10.1002/cnm.1630050204

22. E.d.M.A. Biot. Professor baudouin m. fraeijs de veubeke (1917-1976). https://shellbuckling.com/cv/deveubeke.pdf

23. T.J.R. Hughes, The Finite Element Method: Linear Static and Dynamic Finite Element Analysis (Dover Civil and Mechanical Engineering) (Dover Publications, 2000). URL https://www.xarg.org/ref/a/0486411818/

24. H. Ziegler, in Progress in Solid Mechanics, vol. 4, ed. by I.E. Sneddon, R. Hill (Elsevier North-Holland, 1963), pp. 92-193

25. A. Needleman, J.R. Rice, Acta Metall. 28(10), 1315 (1980)

26. J. Svoboda, I. Turek, Phil. Mag. B 64(6), 749 (1991)

27. F. Parhami, R.M. McMeeking, A.C.F. Cocks, Z. Suo, Mechanics of Materials 31, 43 (1999)

28. K. Hackl, F.D. Fischer, Proc. R. Soc. A 464, 117 (2008)

29. F. Larché, J.W. Cahn, Acta Metall. 21(8), 1051 (1973)

30. S. Boyd, L. Vandenberghe, Convex Optimization. Berichte über verteilte messysteme (Cambridge University Press, 2004)

31. R. Feynman, R. Leighton, M. Sands, E. Hafner, The Feynman Lectures on Physics; Vol. I, vol. 33 (AAPT, 1965)

32. S. Lindsay, Introduction to Nanoscience (OUP Oxford, 2009)

33. M. Ortiz, L. Stainier, Computer Methods in Applied Mechanics and Engineering 171(3), 419 (1999). DOI https://doi.org/10.1016/S0045-7825(98)00219-9

34. C. Miehe, J. Schotte, J. Schroder, Computational Materials Science 16(1), 372 (1999). DOI https://doi.org/10.1016/S0927-0256(99)00080-4

35. M. Tanaka, D. Balzani, J. Schröder, in Innovative Numerical Approaches for Multi-Field and Multi-Scale Problems (Springer International Publishing, 2016), pp. 1-23

36. M. Ortiz, J.B. Martin, International Journal for Numerical Methods in Engineering 28(8), 1839 (1989). DOI $10.1002 /$ nme. 1620280810

37. M. Ortiz, E. Repetto, Journal of the Mechanics and Physics of Solids 47(2), 397 (1999). DOI https://doi.org/10.1016/S0022-5096(97)00096-3

38. D. Balzani, M. Ortiz, International Journal for Numerical Methods in Engineering 12(6), 551 (2012). DOI $10.1002 /$ nme.4351

39. J.M.C. Farias, L. Stainier, E.A. Fancello, Continuum Mechanics and Thermodynamics pp. 1-19 (2019)

40. L. Brassart, L. Stainier, GAMM-Mitteilungen 35(1), 26 (2012)

41. J. Stewart, Calculus (Cengage Learning, 2015)

42. J. Crank, The Mathematics of Diffusion, 2nd edn. (Clarendon Press, Oxford, 1975)

43. J. Bergheau, R. Fortunier, Finite Element Simulation of Heat Transfer. ISTE (Wiley, 2008)

44. A. Latz, J. Zausch, Journal of Power Sources 196(6), 3296 (2011). https://doi.org/10.1016/j.jpowsour.2010.11.088

\section{A Optimality conditions of the functional $\Pi_{\lambda}$}

This appendix gives details about first variations of the functional $\Pi_{\lambda}(9)$, written below with indicial notation:

$$
\Pi_{\lambda}\left[\dot{c}, \lambda_{1}, \lambda_{2}, j_{i}\right]=\int_{\tau}\left[\int_{\Omega}\left(\dot{G}+\chi^{*}+\lambda_{1}\left(\dot{c}+j_{i, i}-r\right)\right) d V+\int_{\partial_{\mu} \Omega} \bar{\mu} j_{i} n_{i} d S+\int_{\partial_{j} \Omega} \lambda_{2}\left(\bar{j}-j_{i} n_{i}\right) d S\right] d t
$$

The term involving the first Lagrange multiplier is first integrated by part, then the divergence theorem is used. One gets

$$
\int_{\Omega} \lambda_{1} j_{i, i} d V=-\int_{\Omega} \lambda_{1, i} j_{i} d V+\int_{\partial_{\mu} \Omega} \lambda_{1} j_{i} n_{i} d S+\int_{\partial_{j} \Omega} \lambda_{1} j_{i} n_{i} d S
$$




\section{A.1 Variation with respect to $j$}

Substituting (48) into (47) gives

$$
\Pi_{\lambda}=\int_{\tau}\left[\int_{\Omega}\left(\dot{G}+\chi^{*}+\lambda_{1}(\dot{c}-r)-\lambda_{1, i} j_{i}\right) d V+\int_{\partial_{\mu} \Omega}\left(\lambda_{1}+\bar{\mu}\right)\left(j_{i} n_{i}\right) d S+\int_{\partial_{j} \Omega} \lambda_{2} \bar{j}+\left(\lambda_{1}-\lambda_{2}\right) j_{i} n_{i} d S\right] d t
$$

Next, taking the variation of the above equation with respect to $j$ leads to

$$
<D_{\boldsymbol{j}} \Pi_{\lambda}, \delta j_{k}>:=\int_{\tau}\left[\int_{\Omega}\left(\frac{\partial \chi^{*}}{\partial j_{k}}-\lambda_{1, k}\right) \delta j_{k} d V+\int_{\partial_{\mu} \Omega}\left(\lambda_{1}+\bar{\mu}\right) n_{k} \delta j_{k} d S-\int_{\partial_{j} \Omega}\left(\lambda_{1}-\lambda_{2}\right) n_{k} \delta j_{k} d S\right] d t
$$

The above first variation should vanish to get the associated stationary conditions. Thanks to the arbitrariness of variations $\delta \boldsymbol{j}$, one gets equations (10)

$$
\begin{array}{rrr}
\frac{\partial \chi^{*}}{\partial j_{k}}-\lambda_{1, k}=0 & \forall \boldsymbol{X} \in \Omega \\
\lambda_{1}+\bar{\mu}=0 & & \forall \boldsymbol{X} \in \partial_{\mu} \Omega \\
\lambda_{1}-\lambda_{2}=0 & \forall \boldsymbol{X} \in \partial_{j} \Omega
\end{array}
$$

Equation (51c) shows that the two Lagrange multipliers are the same variable, i.e. $\lambda_{1}=\lambda_{2}=\lambda$. In addition, that variable is the opposite of the chemical potential according to (51b). Thus we can conclude through equation (51a) that the opposite of the gradient of the chemical potential $-\nabla \mu$ and the flux $j$ are conjugate variables through the dual dissipation potential $\chi^{*}$.

\section{A.2 Variation with respect to $\dot{c}$}

Taking the variation of $(47)$ with respect to $\dot{c}$ gives

$$
<D_{\dot{c}} \Pi_{\lambda}, \delta \dot{c}>:=\int_{\tau}\left[\int_{\Omega}\left(\frac{\partial G}{\partial c}+\lambda_{1}\right) \delta \dot{c} d V\right] d t .
$$

Again the above first variation should vanish to get the associated stationary condition, which leads to (11) thanks to the arbitrariness of $\delta \dot{c}$. As a result, the concentration $c$ and the chemical potential $\mu$ are conjugate variables through the internal energy $G$.

\section{A.3 Variation with respect to $\mu$}

The results shown in equation (12) are immediately obtained given that we have shown that the opposite of the chemical potential $-\mu$ is identical to both Lagrange multipliers $\lambda_{1}$ and $\lambda_{2}$ according to (51c). Thus, by substituting both Lagrange multipliers by the opposite of the chemical potential in (47) and applying the variation with respect to the chemical potential directly leads to equation (12). 